\title{
Thinking ahead: the case for motor imagery in prospective judgements of prehension
}

\author{
Scott H. Johnson* \\ Dartmouth College, Center for Cognitive Neuroscience, 6162 Moore Hall, Hanover, \\ NH 03755-3569, USA
}

Received 8 October 1998; received in revised form 11 May 1999; accepted 17 September 1999

\begin{abstract}
How similar are judgements concerning how we expect to perform an action, to how we actually behave? The veracity of such prospective action judgements, and the mechanisms by which they are computed, was explored in a series of tasks that involved either grasping (MC conditions) or thinking about grasping (PJ conditions) a dowel presented in various orientations. PJs concerning limits of comfortable hand supination and pronation when turning a dowel in the picture plane were highly consistent with values obtained during actual hand rotation (Exp. 1). The same was true for judgements regarding the level of awkwardness involved in adopting a prescribed grip (e.g. overhand with right hand) for dowels in various picture plane orientations (Exp. 2). When allowed to select the most natural grip (overhand versus underhand) or hand (left versus right) for engaging dowels in these orientations, subjects preferred virtually identical responses in both PJ and MC conditions. In both instances, they consistently chose the least awkward response options. As would be expected for actual movements, PJs involving awkward hand postures had longer response times (RTs), and were less accurate. Likewise, latencies for both grip and hand judgements tended to increase as a function of the angular distance between the current positions of subjects' hands, and the orientation of the chosen posture. Together, these findings are consistent with a the hypothesis that PJs involve mentally simulated actions, or motor imagery. These results suggest that motor imagery does not depend on the existence of a completed premotor plan (Jeannerod, 1994), but may instead be involved in the planning process itself. A provisional model for the involvement of imagery in motor planning is outlined, as are a set of criteria for evaluating claims of the involvement of motor imagery in problem solving. (C) 2000 Elsevier Science B.V. All rights reserved.
\end{abstract}

Keywords: Motor imagery; Prospective judgements; Prehension

\footnotetext{
* Tel.: +1-603-646-0040 (office); +1-603-646-1180 (secretary); +1-413-549-6206 (home); fax: +1603-646-1181.

E-mail address: scott.h.johnson@dartmouth.edu (S.H. Johnson)
}

0010-0277/00/\$ - see front matter (C) 2000 Elsevier Science B.V. All rights reserved. PII: S0010-0277(99)00063-3 
The ability to anticipate the consequences of potential actions and modify our responses accordingly has long been considered a fundamental aspect of intelligent behavior (e.g. James, 1890). But, just how accurate is the ability to forecast our actions? In the domain of motor control, previous work has shown that actors tend to misjudge the limits of their own physical capabilities to varying degrees (e.g. Carello, Grosofsky, Reichel, Solomon \& Turvey, 1989; Heft, 1993; Jiang \& Mark, 1994; Mark, 1995; Mark \& Vogele, 1988; Mark, Nemeth, Gardner, Dainoff, Paasche, Duffy \& Grant, 1997, Exp. 1; Robinovitch \& Cronin, 1997). For instance, when asked to judge the maximum distance at which an object can be reached by simply extending one arm without involving the shoulder or torso, subjects' estimates consistently fell short of their absolute critical boundaries (Warren, 1984), as dictated by their physical limitations (e.g. Mark et al., 1997, Exp. 1, Task 1). Advocates of the direct perception approach have interpreted these and similar results as suggesting that, when perceiving objects' affordances, actors tend to err on the conservative side in order to avoid initiating movements that cannot be completed (e.g. Jiang \& Mark, 1994). However, such underestimates may simply reflect the fact that subjects generally avoid performing actions that approach their physical limitations, and therefore have little experience with such extremes (cf. Mark et al., 1997, p. 1376). Instead of stretching the arm to its maximum, for example, we tend to reach more distant objects by rotating the shoulder, and/or bending at the waist; a more comfortable, less awkward alternative. As a result, the consequences of such ungainly movements may be more difficult to recall, or to mentally simulate. When allowed to choose freely between these options, subjects tend to shift their mode of responding at a point that is consistently less than their absolute critical boundary (e.g. Cutting, 1982) - a preferred critical boundary (Mark, 1995). These observations suggest that if presented with a choice between two or more options, subjects' prospective action judgements may in fact be much more consistent with their actual response preferences than has previously been thought (e.g. Mark et al., 1997).

This project focused on two questions. First, when given the opportunity to choose the response that seems appropriate under a given set of task constraints, how similar are PJs to actions? Under these more natural circumstances, do subjects still display the tendency to underestimate the limits of their actual performances, or are PJs veridical? Second, how are PJs about actions undertaken? Do subjects somehow directly perceive the affordances of the task (e.g. Gibson, 1979), or do they evaluate candidate actions by constructing internal representations? Indeed, there is considerable evidence from the motor planning literature that subjects do use premotor representations when selecting among movement options (e.g. Klatzky, McCloskey, Doherty, Pelligrino \& Smith, 1987; Klatzky, Pelligrino, McCloskey \& Lederman, 1993; Marteniuk, MacKenzie, Jeannerod, Athenes \& Dugas, 1987; Rosenbaum \& Jorgenson, 1990; Rosenbaum, Vaughan, Barnes \& Jorgensen, 1992; Stelmach, Castiello \& Jeannerod, 1994). Consistent with this representational stance is the possibility that prospective action judgements may involve internally simulated movements, or motor imagery. Jeannerod $(1994,1995)$ has suggested that motor imagery is the conscious experience of a normally non-conscious premotor 
plan. From this perspective motor imagery is experienced when execution of a fully formed premotor plan is inhibited. However, it is also possible that motor imagery may actually be involved during computation of the premotor plan. According to this imagery as planning hypothesis, solving the ill-posed problem of movement selection (e.g. Bernstein, 1967; Rosenbaum, 1991) involves mentally simulating candidate response options in order to evaluate their consequences in advance of their execution.

Like visual imagery, motor imagery is thought to involve operations performed on analog representations. Unlike visual imagery, however, these somatomotor representations are thought to make explicit the biomechanical constraints of the human body (e.g. Jeannerod, 1994; Johnson, 1998; Johnson, Corballis \& Gazzaniga, 1999; Parsons, 1987a,b, 1994). Consequently, if prospective action judgements involve motor imagery, then responses should be consistent with actual movements selected under comparable circumstances. Furthermore, the amount of time required to make such decisions should be affected by variables known to influence the timecourse of actual movements, namely awkwardness (e.g. Parsons, 1994) and extent (e.g. Decety \& Jeannerod, 1995; Decety, Jeannerod, \& Prablanc, 1989; Fitts, 1954).

\section{Overview}

In order to address these issues, pairs of tasks were created in which subjects were required to either (a) actually grasp a visually presented dowel or (b) judge how they would grasp a dowel under comparable circumstances. The underlying logic was to manipulate a perceptual variable known to affect grip selection - the orientation of a manipulandum (e.g. Rosenbaum \& Jorgenson, 1990), and compare the consequences on both prehension and prospective action judgements.

Experiment 1 established the comfortable range of motion for hand (i.e. forearm) rotation, the primary biomechanical constraint on grip selection in these tasks, and examined how accurately subjects could judge this range in the absence of overt movements. Experiments 2- 4 are divided into two sections. Experiments labeled ' a' required subjects to overtly reach for the manipulandum, while experiments labeled ' $b$ ' required subjects to make PJs about how they would reach, while keeping their hands stationary. Experiments $2 \mathrm{a}$ and $2 \mathrm{~b}$ compared ratings of perceived awkwardness when both a particular grip (overhand versus underhand) and response hand (left versus right) were designated in advance. Experiments $3 \mathrm{a}$ and $3 \mathrm{~b}$ compared subjects' preferences when selecting a grip with a prescribed hand. Experiments $4 \mathrm{a}$ and $4 \mathrm{~b}$ compared preferences when selecting a response hand in order to adopt a designated grip.

\section{Experiment 1: limits of comfortable hand rotation}

One primary limitation on the ability to grasp an object in different orientations is biomechanical constraints on the range of forearm rotation. These limits can be 
demonstrated by extending both arms and giving the " thumbs up" signal. Rotating your thumbs medially, as if giving the " thumbs down" signal, involves forearm pronation. In contrast, rotating the thumbs laterally, as if giving directions to " go left" or " go right," involves forearm supination. This demonstration makes it clear that the range of motion of forearm rotation depends on the direction of movement; that is, forearm pronation has a considerably larger range of motion than does supination. Furthermore, because they are essentially mirror reflections of one another, the biomechanical constraints of the two arms are approximately $180^{\circ}$ out of phase (e.g. American Academy of Orthopaedic Surgery, 1965, cited in MacKenzie \& Iberall, 1994; Parsons, 1994).

Subjects in Exp. 1 performed two tasks. In the Prospective Judgement (PJ) condition, subjects were told to estimate how far they could comfortably rotate their left and right hands if they were grasping a rotating dowel in a power grip (i.e. as one would grap the handle of a hammer). In the Motor Control (MC) condition, they actively rotated the handle until they reached their perceived comfort limits. Of interest was how accurately subjects could estimate the comfortable range of hand rotation in the absence of visual and proprioceptive feedback. As noted above, earlier work suggests that PJs consistently underestimate critical boundaries (e.g. Jiang \& Mark, 1994; Warren, 1984). However, because subjects typically avoid such extreme postures (e.g. Cutting, 1982), the tendency to underestimate their physical capabilities may be attributable to lack of experience, rather than an inherent perceptual bias. If so, then PJs concerning limits of comfortable hand rotation should be considerably more veridical.

\subsection{Method}

Subjects. Eight (seven right-handed and one left-handed) subjects voluntarily participated in a single, 20 min testing session. Subjects did not take part in any related experiments, and were naive to the hypotheses being evaluated.

Stimuli and apparatus. The stimulus was a 1 inch diameter wooden dowel measuring 6 inches in length. The dowel subtended approximately $2.6^{\circ}$ by $10.8^{\circ}$ of visual angle when viewed from $50 \mathrm{~cm}$. Half of the dowel was colored pink, and the other half was colored tan. The dowel was suspended in the center of a wooden box that was open at the front. The box was painted flat black. The front side of the box was open so as not to obstruct reaching movements, and measured 24 inches by 24 inches. The dowel was attached to an axle at both ends. The rear of the axle protruded through the back wall of the box, allowing the experimenter to accurately determine the orientation of the stimulus by observing the relationship between a pointer attached to the axle and degrees mapped on the back of the box. From the subject's perspective, only the stimulus and the edges of the surrounding box were visible.

Subjects were seated in front of the apparatus with their hands resting palms down on designated locations on the table surface.

Procedure. In order to eliminate the possibility that subjects would rely on memories of responses from the MC task, the PJ condition was always performed 
first. Each condition consisted of one practise block, and seven experimental blocks. A block included four trials: supination and pronation with each hand. Trial order was counterbalanced across blocks. At the beginning of each trial, the experimenter positioned the dowel in a vertical orientation with the pink end pointing upward.

In the PJ condition, subjects were instructed to estimate how far the dowel could rotate before reaching the limits of their comfortable range of motion if they were grasping it in a power grip with the thumbside of their hand on the pink end. Through demonstration the experimenter explained that they should base their judgements on rotations of both the forearm and shoulder, but not movements of the torso. Subjects were instructed to keep their hands still while deciding. At the beginning of each trial, subjects were informed which hand to base their decision on, and the experimenter began rotating the dowel in either the clockwise or counter-clockwise direction at a rate of approximately $30 \%$ s. Subjects were instructed to respond by saying ' stop' when the dowel reached an angle that would be the maximum limit of comfortable hand rotation. The experimenter then recorded the angle from the dial on the rear of the apparatus and reoriented the dowel to begin the next trial.

In the MC condition, subjects were instructed to actually grasp the dowel with thumbside of the hand toward the pink end, and turn it until they reached the limits of their comfortable range of motion. It was emphasized that subjects should keep their torsos stationary, but could rotate both their forearms and shoulders. At the beginning of each trial they were told which hand to use and in which direction to rotate the dowel (i.e, supination versus pronation). Maximum angles were then recorded and the dowel was returned to the starting orientation.

\subsection{Results and discussion}

Data were collapsed across trials to obtain mean angles of maximum supination and pronation for each subject using each hand. As shown in Fig. 1, performances in the PJ and MC conditions were remarkably consistent. A repeated measures ANOVA found no significant difference between PJ and MC conditions or between left and right hands, $F<1.0$ in both cases. In each condition subjects showed a substantially greater range of motion for pronation versus supination, $F(1,7)=40.8, P<0.0001, M S e=1787$. In contrast to earlier work, there was no evidence that PJs underestimate actual ranges of motion (e.g. Carello et al., 1989; Heft, 1993; Jiang \& Mark, 1994; Mark, 1995; Mark, et al., 1997; Warren, 1984, Exp. 1; Robinovitch \& Cronin, 1997). It seems unlikely that greater accuracy in the present task is attributable to it being substantially less difficult than those used in these previous experiments. Warren (1984), for example, had subjects judge the maximal distance at which a target could be reached solely by extending one arm, an action that also involves movements of the lower and upper arms. Instead, the present task involves judgements based on complex, multi-joint knowledge of the ranges of motion of the shoulder, elbow, and wrist. Veridical judgements in the present task instead suggest that prospective action judgements involve some form 


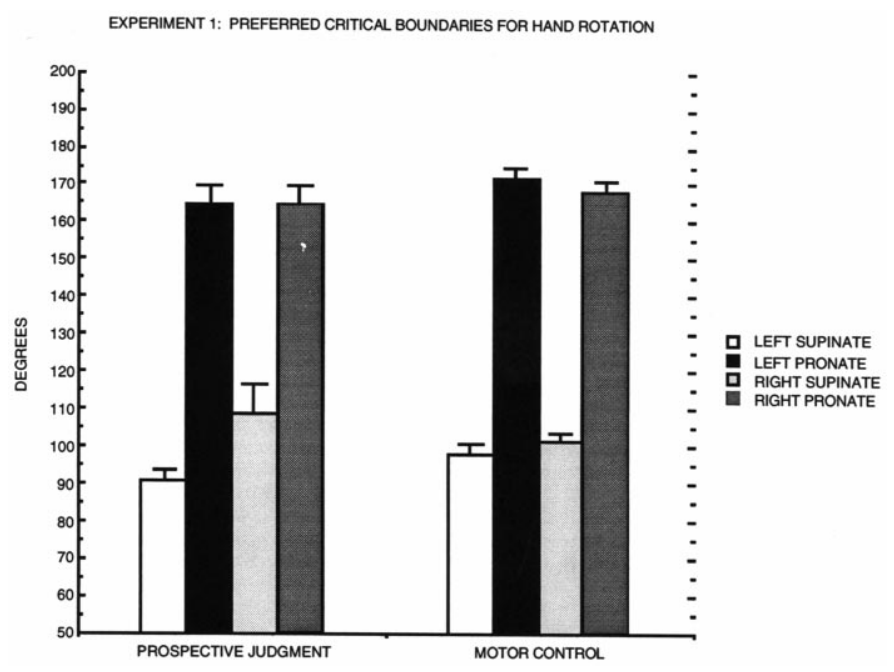

Fig. 1. Comfortable range of motion in motor control and prospectuve judgements. Prospective judgements of limits on the comfortable range of hand rotation are highly similar to those in MC. In both conditions, the range of motion is greater for pronation than supination. Errors bars represent standard errors of the means.

of experience-dependent processing. The following experiments attempt to understand the precise nature of these processes.

\section{Experiment 2: perceived awkwardness}

Experiment 1 showed that subjects can accurately judge limits of comfortable hand rotation in the absence of overt movements and associated sensory feedback. This observation suggests that mechanisms involved in PJs are highly sensitive to the biomechanical demands, or awkwardness, of familiar movements. Experiment 2 evaluated this possibility directly.

In Exp. 2a, subjects were instructed to grasp a visually presented dowel so that the thumbside of their hand was always toward a designated end, and then rate how awkward the resulting grip felt. In Exp. 2b, subjects were instructed to judge how awkward it would be to grasp a dowel under the same conditions, but without actually moving. In this later study, and in all subsequent PJ tasks, dowels were photo-realistic, computer generated images. This change provided more precise control over the timing of stimulus presentations, and allowed for accurate RT recording. Additionally, graphically rendered stimuli served as a constant reminder to subjects that they were not allowed to actually reach during this condition.

To the extent that processes involved in prospective awkwardness judgements are sensitive to biomechanical demands of hand rotation, similar awkwardness ratings were expected in both Exps. 2a and 2b. Further, if processes involved in PJs are 
experience dependent, then estimates should be less veridical when based on grips involving more extreme, and therefore less familiar, hand orientations.

\subsection{Experiment 2a: motor control}

\subsubsection{Method}

A group of 6 right-handed undergraduates from the University of Massachusetts participated in a single $30 \mathrm{~min}$ testing session for $\$ 5.00$. These subjects had not participated in any related experiments, and were naive to the hypotheses being evaluated. The stimulus and apparatus were similar to those used in Exp. 1.

Subjects were told that their task would be to reach for the center of the dowel using a power grip such that the thumbside of the designated response hand would always be toward the designated end (either pink or tan). Each subject participated in three blocks of trials using their right hand, and three blocks using their left hand. Order of both response hand and designated end of the stimulus was counterbalanced across blocks. Order of the blocks was counterbalanced across subjects. Subjects were instructed to begin their reach as soon as the stimulus became illuminated, and to immediately rate the awkwardness of the acquired grip on a scale ranging from one (not awkward) to five (highly awkward). Awkwardness ratings were made verbally while the object was still being grasped, and subjects were instructed to use the entire rating scale.

At the beginning of a block of trials, subjects were asked to place the designated response hand palm down on a location marked on the table in front of the apparatus. The non-responding arm was bent $90^{\circ}$ at the elbow, and rested on a designated location at the edge of the table. Over the course of each block, the stimulus occurred randomly in 24 different orientations: $15^{\circ}$ increments. These orientations are illustrated in Fig. 2. Because the hands are mirror images of one another, stimulus orientation is expressed according to the number of degrees of rotation from the neutral posture $\left(0^{\circ}\right)$ moving in the direction of pronation, clockwise for the left hand and counter-clockwise for the right. This convention, referred to as relative hand orientation, is used throughout the rest of the paper.

At the beginning of each trial the subject was verbally instructed to prepare to reach. After a delay of approximately $1 \mathrm{~s}$ the experimenter threw a switch illuminating the dowel. The subject then reached, and verbally rated how awkward it felt. The experimenter recorded the response and the subject was instructed to release the stimulus and return to the starting position. The experimenter then rotated the dowel to the next orientation, and the subsequent trial was initiated.

\subsubsection{Results}

For each hand, awkwardness ratings involving identical stimulus orientations were combined across blocks in which the designated end of the stimulus was pink or tan. For instance, awkwardness ratings were combined from trials where subjects were instructed to grasp the pink end for the $0^{\circ}$ stimulus and the tan end for the $180^{\circ}$ stimulus (see Fig. 2). As shown in Fig. 3 (panel A), when expressed as a function of relative hand orientation perceptions of awkwardness vary in a nearly 


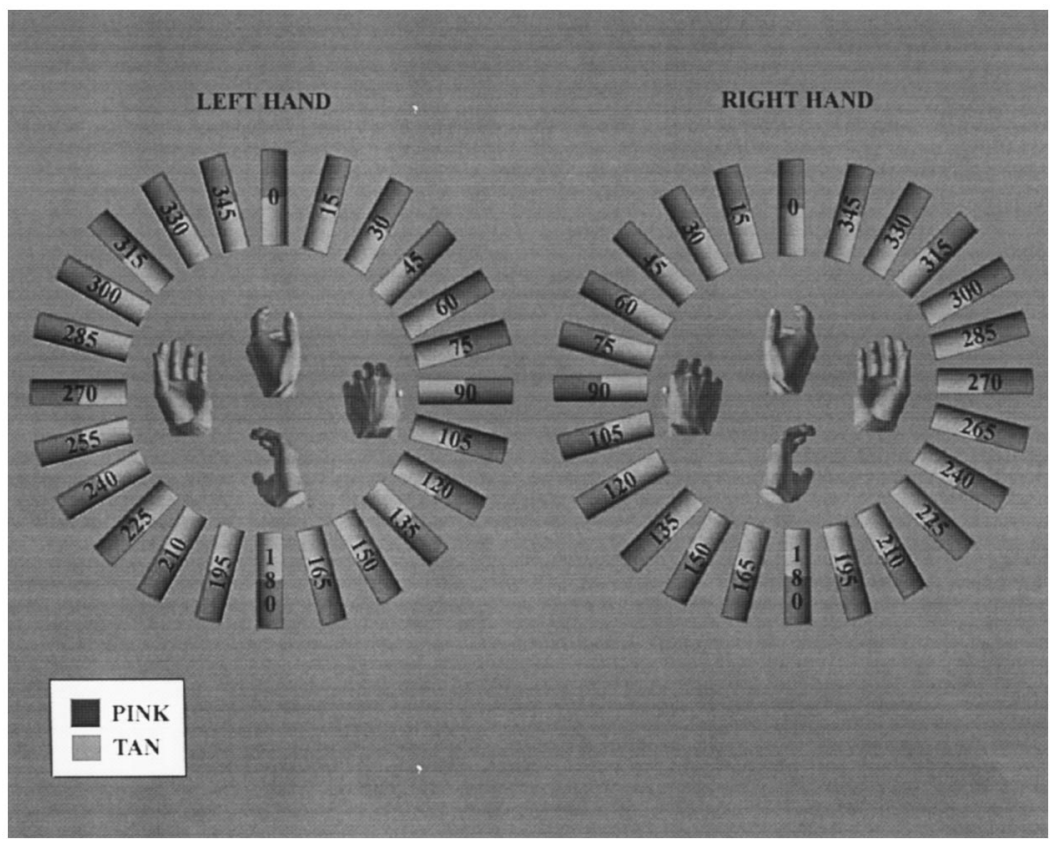

Fig. 2. The 24 stimulus orientations evaluated in Exps. 2- 4. Note that in all figures, $0^{\circ}$ of orientation represents the stimulus positioned vertically with the pink end pointing upward. Because the joint constraints of the two hands are mirror images, stimulus orientation is plotted as relative hand orientation with the dowel advancing in a clockwise direction for the left hand and counter-clockwise for the right hand.

identical fashion for each hand, $(R=0.98, F(1,22)=683.0, \quad P<0.0001$, $M S e=0.04)$. These functions reflect a nearly perfect mirror symmetry between joint constraints on rotations of the two hands.

Beginning at $15^{\circ}$ both hands were pronating, and awkwardness increased gradually beginning at approximately $90^{\circ}$. At approximately $170^{\circ}$, both hands reached the limits of comfortable pronation, as estimated in the MC condition of Exp. 1. (see Fig. 1). However, subjects chose to maintain the pronated (i.e. overhand) grip because the supinated (i.e. underhand) option was still biomechanically impossible. Maximum awkwardness for the left and right hands was reached at $225^{\circ}$. These peaks represent the absolute critical boundaries at which subjects reached the biomechanical limits of pronation and were forced to adopt a supinated grip in order to keep their thumb on the designated end of the stimulus. Hand orientations immediately after this transition still exceed the critical boundaries for comfortable supination, as estimated in the MC condition of Exp. 1, but are physically possible. Perception of awkwardness then diminished as the hands rotated back toward the neutral $0^{\circ}$ position.

In sum, Exp. 2a demonstrated that the relationship between relative hand orientation and perceptions of grip awkwardness are nearly identical for the left and right 

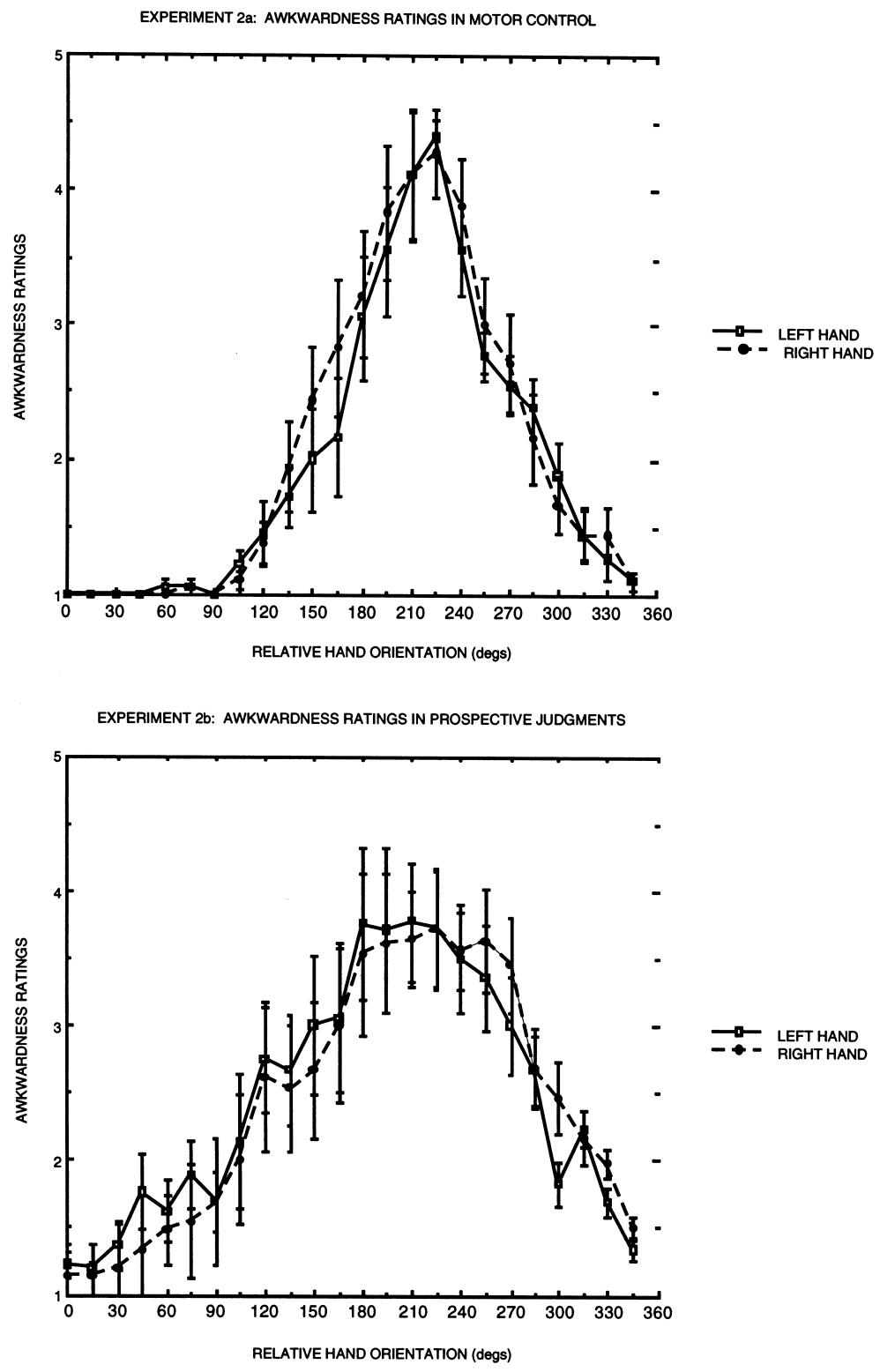

Fig. 3. Relationship between awkwardness ratings and relative hand orientations. Panel A. Because their joint constraints are mirror reflections of one another, the relationship between perceived awkwardness in $\mathrm{MC}$ and relative hand orientation is virtually identical for left and right hands. Error bars in this and all subsequent figures reflect the standard error of the means computed across subjects. Panel B. Similar to $\mathrm{MC}$, PJs of awkwardness accurately reflect the opposing biomechanical constraints on rotations of the left and right hands. 
hands. As expected, subjects perceived grips that approach their critical boundaries for supination or pronation as considerably more awkward than those in the middle of their comfortable ranges of motion.

\subsection{Experiment $2 b$ : prospective judgements}

Experiment $2 \mathrm{~b}$ focused on two questions. First, how accurate would subjects be if asked to evaluate awkwardness without actually reaching? If prospective awkwardness judgements are sensitive to biomechanical constraints, then estimates based on the left and right hands should be similarly affected by changes in relative hand orientation, and should peak at approximately $225^{\circ}$ (Fig. 3, panel A). Accurate estimates of awkwardness would indicate that such information is potentially available to premovement planning processes.

Second, how are PJs of awkwardness accomplished? It is well-known that awkward movements encounter more biomechanical resistance, and therefore take longer to perform, and there is reason to believe that the same is true for imagined movements (e.g. Parsons, 1994). If PJs of awkwardness involve motor imagery, then grips perceived as being more awkward should also take more time to evaluate.

\subsubsection{Method}

Six University of Massachusetts undergraduate students participated in a single 40 min testing session for course credit. None of these subjects had participated in any related experiments, and all were naive to the hypotheses being evaluated. The tasks were automated by using graphically rendered stimuli. As in Exps. 1 and 2a, stimuli subtended approximately $2.6^{\circ}$ by $10.8^{\circ}$ of visual angle when viewed from $50 \mathrm{~cm}$. Shading and reflectance were used to create the illusion of being threedimensional. Half of the stimulus dowel was colored pink and the other half was colored tan. Twenty-four versions of the dowel were created by rotating the stimulus in $15^{\circ}$ increments around a full circle (refer to Fig. 2). Stimuli were presented, and responses were recorded, using a Macintosh Quadra 840av equipped with a 20 inch color monitor and vocal response key.

Procedure. At the beginning of the experiment subjects were allowed to read a brief description of the procedure from the computer screen. The experimenter then reiterated the instructions emphasizing that subjects should respond as soon as they had made their decisions, and should not move their hands. To ensure compliance with the instructions not to move, the position of subjects' hands was monitored by the experimenter throughout the study. No references to using imagery in order to solve this task were made.

The experiment consisted of 14 blocks. Response hand and the designated end of the dowel (i.e. pink or tan) were counterbalanced across blocks. Order of the blocks was counterbalanced across subjects. Within each block, stimuli appeared randomly in each of the 24 different orientations (see Fig. 2). Subjects were given a self-timed rest break following the seventh block.

At the beginning of each block, a message appeared designating which hand awkwardness judgements would be based on, and which end of the dowel the 
thumb would be toward. To ensure that subjects remembered which hand they were to base their judgements on, the designated response hand was positioned palm down on a marker in front of the subject. The non-responding arm was bent $90^{\circ}$ at the elbow, and rested on a line marked along the edge of the table.

Subjects were instructed to evaluate how awkward it would be to grasp the dowel in the center using a power grip such that the thumbside of their response hand was toward the designated end (i.e. pink or tan). A model dowel was used by the experimenter to demonstrate the correct power grip. Subjects verbally rated how awkward grips would be on a scale ranging from one (not awkward) to five (very awkward). They were encouraged to use the entire rating scale.

Each trial began with a $1000 \mathrm{~ms}$ " Ready!" signal, which was immediately replaced by a fixation point lasting for $500 \mathrm{~ms}$. The stimulus dowel appeared immediately after the offset of the fixation point, and remained visible until subjects responded, or until $3000 \mathrm{~ms}$ elapsed. The response timer was initiated when the stimulus became visible, and was terminated by the subject's vocal response into a microphone suspended from the ceiling. At the end of each trial, the experimenter entered the response by typing the designated digit into the keyboard. To ensure accuracy, the digit had to be entered twice. The second entry initiated the subsequent trial.

\subsubsection{Results}

Awkwardness ratings. As described above for Exp. 2a, for both the left and right hands awkwardness ratings involving similarly orientated stimuli were combined across blocks in which the designated end was pink or tan. Fig. 3 (panel B) shows that the relationship between awkwardness ratings and relative hand orientation was nearly identical for the two hands, $(R=0.96, F(1,22)=288, P<0.0001$, $M S e=0.06$ ). Despite the fact that no actual movements were involved, awkwardness judgements accurately reflect the mirror image joint constraints of the two hands. Orientations at which peak awkwardness values were achieved for the left $\left(M=188^{\circ}, S D=53^{\circ}\right)$ and right $\left(M=184^{\circ}, \mathrm{SD}=35^{\circ}\right)$ hands were not significantly different, $T(5)=0.48, P=0.65$.

Comparison of awkwardness ratings in Exps. $2 a$ and $2 b$. Prospective awkwardness judgements (Exp. 2b) were highly correlated with those obtained when subjects actually grasped the stimulus (Exp. 2a), $R=0.92, F(1,46)=252, P<0.001$, $M S e=0.127$ (cf. Fig. 3, panels A and B). In both tasks, awkwardness values peaked at approximately $225^{\circ}$ the point at which subjects in Exp. 2a transitioned to a supinated grip. PJs, however, yielded slightly lower peak awkwardness ratings, $T(11)=-4.0, P=0.002$.

As expected if experience-dependent mechanisms are involved, PJs were more accurate, i.e. more similar to ratings for actual prehension, for those hand orientations that were perceived as less awkward. This was true for both the left $(R=0.94$, $F(1,22)=154, P<0.0001, M S e=0.17)$ and right $(R=0.91, F(1,22)=106.3$, $P<0.0001, M S e=0.21)$ hands. Fig. 4 depicts differences between awkwardness ratings in Exps. $2 \mathrm{a}$ and $2 \mathrm{~b}$ as a function of relative hand orientation and response hand. In general, these differences are small, however, as indicated by the positive 


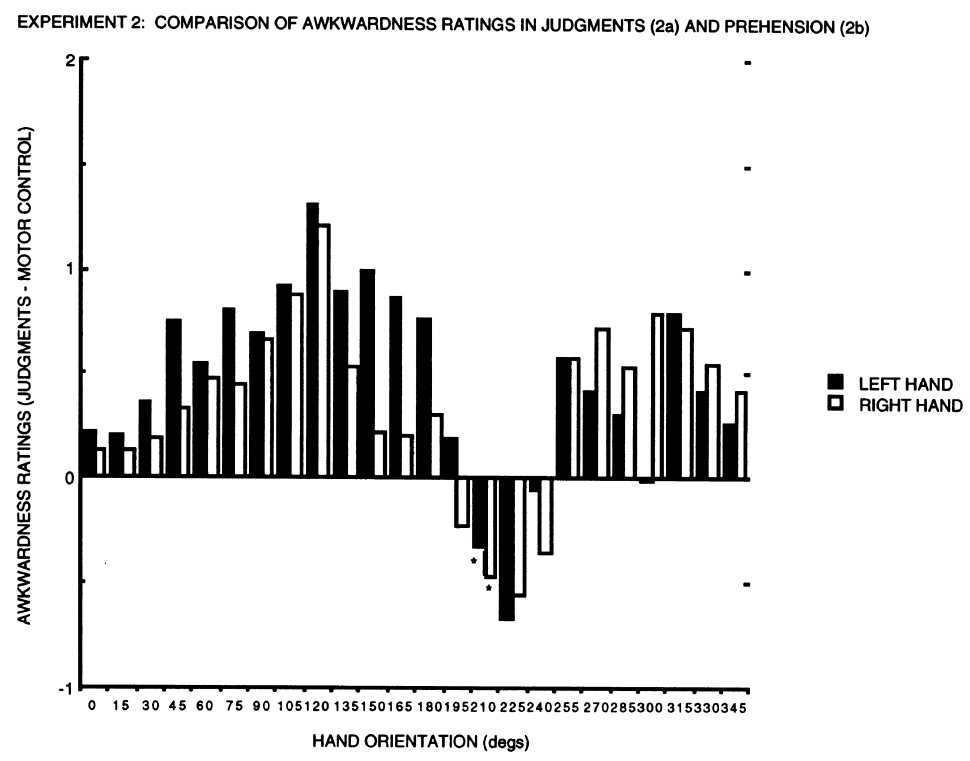

Fig. 4. Differences between awkwardness ratings in MC (Exp. 2a) and PJs (Exp. 2b). Positive values represent overestimates in awkwardness judgements, and negative values represent underestimates. Asterisks indicate hand orientations perceived as maximally awkward in Exp. 2a. Note the tendency for judgements to underestimate the highly awkward postures.

values, there was a tendency for PJs to overestimate at orientations perceived as being of low to moderate awkwardness $\left(0^{\circ}-180^{\circ}\right.$ and $\left.255^{\circ}-345^{\circ}\right)$. In contrast, for those orientations perceived as being maximally awkward, there was a tendency for PJs to underestimate the degree of awkwardness (Fig. 4). This finding is inconsistent with the hypothesis that PJs err on the conservative side in order to provide a margin of safety when evaluating potential actions (e.g. Jiang and Mark, 1994). If so, then one would expect prospective awkwardness ratings to over- rather than under-estimate the difficulty of highly awkward postures.

Response times. Responses greater than $3000 \mathrm{~ms}$ or less than $300 \mathrm{~ms}$ were eliminated as outliers (approximately 5\% of the total). Mean RTs were then calculated separately for each subject using the left and right hands at each stimulus orientation. As expected if processes involved in PJs are sensitive to biomechanical constraints, RTs for both hands varied similarly as a function of relative hand orientation, $R=0.58, F(1,22)=11.0, P=0.003, M S e=18653$. As depicted in Fig. 5, this correlation reflects the degree to which the left and right hand RT profiles are mirror images of one another.

Consistent with the hypothesis that imagined reaching is used in making PJs, there was a significant positive correlation between rated awkwardness and the time required to make the rating, $R=0.36, F(1,46)=6.8, P=0.01, M S e=0.72$. That is, awkward grips tended to require more time to evaluate than did less awkward grips. 


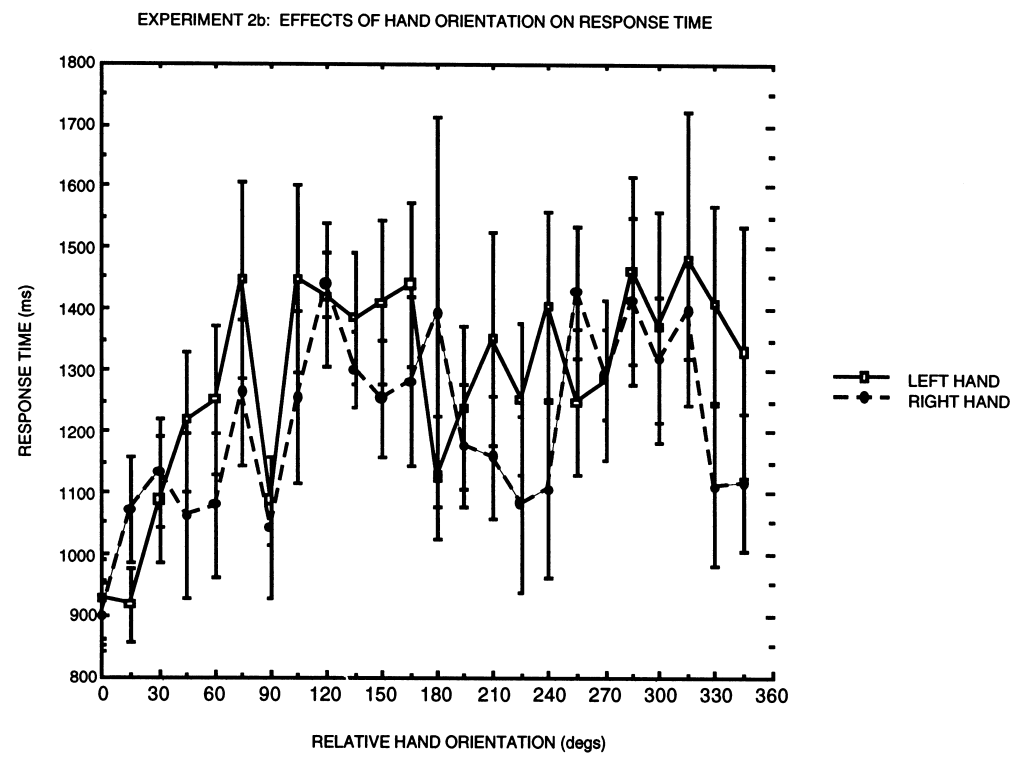

Fig. 5. Relationship between relative hand orientation and response time. Response times for PJs based on the left and right hands vary similarly as a function of relative hand orientation. In general, more awkward grips took longer to evaluate.

\subsubsection{Discussion}

Subjects' prospective awkwardness ratings were generally quite consistent with estimates provided by a separate group during actual prehension. Although constrained to grasping the dowel with a particular hand so that their thumbs would be on a designated end, subjects in both experiments were free to select either overhand or underhand grips. In MC (Exp. 2a), peak awkwardness values occurred at preferred critical boundaries where subjects switched from an awkward to a less awkward grip (see also Mark et al., 1997). Corresponding changes in prospective awkwardness judgements (Exp. 2b) suggest that subjects may have ' mentally' switched grips at comparable stimulus orientations while making these decisions. In other words, preferred critical boundaries in both prehension and PJs may be quite similar. This possibility will be explored further in Exps. 3 and 4.

The accuracy of PJs did tend to diminish as grips became more awkward, as rated in MC. For most orientations, there was a tendency for judgements to overestimate actual awkwardness. However, for orientations that were rated as most awkward, PJs tended to underestimate perceived awkwardness. The fact that the amount of time required to make PJs of awkwardness also tended to increase as a function of perceived awkwardness, indicates that this pattern cannot be attributed to a simple speed- accuracy tradeoff. One possibility is that motor imagery is involved in making these judgements, and that more awkward postures may be more difficult to accurately simulate. This might be expected given that subjects undoubtedly have less experience grasping objects with their hands in extreme orientations. This 
interpretation is consistent with previous work by Parsons (1994), suggesting that awkward postures are more difficult to imagine as well as adopt.

Further support for the motor imagery hypothesis was found in RT data. There was a tendency for RTs to increase as a function of rated awkwardness, suggesting that awkward postures may also require more time to simulate. If subjects were performing an analog simulation of reaching movements, then response latencies should also increase as a function of the extent of the movement, i.e. stimuli requiring greater hand rotation should take longer to evaluate. To test this hypothesis, RTs were regressed onto a variable that captured angular distance of each stimulus orientation from the fastest orientation with the left and right hands $\left(15^{\circ}\right.$ for the left hand, $0^{\circ}$ for the right hand). It is worth noting that these RT minima did not coincide with the actual physical locations of subjects' left and right hands, which were instead in the same plane as the $90^{\circ}$ stimuli. The direction of hand rotation was determined based on the location of critical boundaries in awkwardness ratings during actual prehension $\left(225^{\circ}\right)$. However, because subjects were free to choose either an underhand (supinated) or overhand (pronated) grip in this study, these boundaries are only estimates based on the orientations where awkwardness ratings peaked. Consequently, the angular distance variable is only an approximation. Nevertheless, RTs did tend to increase as a function of angular distance, $(R=0.40, F(1,46)=8.4, P=0.006, M S e=21756$. The rate of this transformation, as determined by the slope of the best-fitting linear function through these points, would be approximately $886 \%$ s. This relationship is more rigorously evaluated in Exps. 3 and 4 where critical boundaries in movement selection are well defined.

\section{Experiment 3: grip selection}

Previous studies have shown that perceptions of awkwardness are highly predictive of movement selection in prehension (e.g. Mark et al., 1997; Rosenbaum et al., 1992, Exp. 1a). Having established that subjects could accurately evaluate awkwardness without actively engaging in reaches, it was of interest to determine whether these estimates were also related to movement selection in PJs. In Exp. 3 subjects were presented with a dowel in a variety of different orientations in the picture plane and allowed to choose the most natural grip, overhand (pronated) or underhand (supinated). In Exp. 3a subjects actually reached for the stimulus (MC), and in Exp. $3 b$ subjects judged how they would reach in the absence of overt movements (PJ).

Given the opportunity to select the most appropriate action, it was expected that subjects' prospective grip judgements would be highly similar to their actual responses. In both instances, it was predicted that subjects would prefer grips that minimized awkwardness. If so then hand orientations at which subjects switch from one grip option to another, their preferred critical boundaries, should coincide with increases in awkwardness of the initial grip, yet occur before those hand orientations perceived as maximally awkward (i.e. critical boundaries) are reached. Conse- 
quently, functions relating grip preferences to relative hand orientation should also be highly similar for the two hands, reflecting the fact that the biomechanical constraints of the two arms are inversely related (MacKenzie \& Iberall, 1994; American Academy of Orthopaedic Surgeons, 1965, cited in Parsons, 1994). If grip selection involves evaluations of awkwardness, then based on the findings of Exp. 2 PJs should be less accurate at orientations surrounding preferred critical boundaries, i.e. those orientations that would involve adopting the most awkward grips.

Finally, if prospective grip judgements involve motor imagery, then RTs for grip selection at specific orientations should increase as a function how awkward the chosen grip would be to actually adopt (Exp. 2a). In other words, as suggested in Exp. $2 b$, it should take more time to mentally simulate awkward actions.

\subsection{Experiment 3a: motor control}

\subsubsection{Method}

Six right-handed, University of Massachusetts undergraduates participated in a single 30 min testing session for $\$ 5.00$. None of the subjects were involved in any related experiments, or were aware of the hypotheses under investigation. The stimuli and apparatus were identical to those employed in Exp. 2a. Each subject participated in three blocks of trials using their right hand, and three blocks using their left hand. Order of the blocks (left versus right hand) was counterbalanced across subjects. Stimulus orientation was randomized within each block.

Subjects were instructed to use the designated response hand to grasp the dowel in the center using a power grip, and were told that they were free to choose either an overhand (pronated) or underhand (supinated) grip, as demonstrated by the experimenter. Once the reach on a given trial was completed, the experimenter manually recorded whether the thumbside of subject's hand was toward the pink or tan end of the stimulus, by observing their reflections in a mirror.

\subsubsection{Results}

The probability of selecting the " pink" grip was calculated separately for each subject using each hand for each stimulus orientation. As predicted, subjects' grip preferences were highly consistent with awkwardness ratings obtained in Exp. 2a. That is, there was a strong negative correlation between the probability of selecting the pink grip for a stimulus at a particular orientation, and rated awkwardness of that particular grip $(R=-0.87, F(1,46)=147.4, P<0.001, M S e=0.049$.

Consistent with expectations, Fig. 6 (panel A) shows that functions relating grip preferences to relative hand orientation were virtually identical for the two hands, $R=0.99, F(1,22)=786.3, P<0.0001, M S e=0.006$. Again, preferred critical boundaries, or points of transition between grip preferences, can be estimated by identifying the relative hand orientations at which the probability of selecting one or the other grip first exceeds 0.50 . Subjects favored the pink grip until approximately $130^{\circ}$, which is considerably less than the limits on comfortable pronation (approx. $170^{\circ}$, Fig. 1), or peak awkwardness $\left(225^{\circ}\right.$, Fig. 3, panel A). At this point, a preferred 

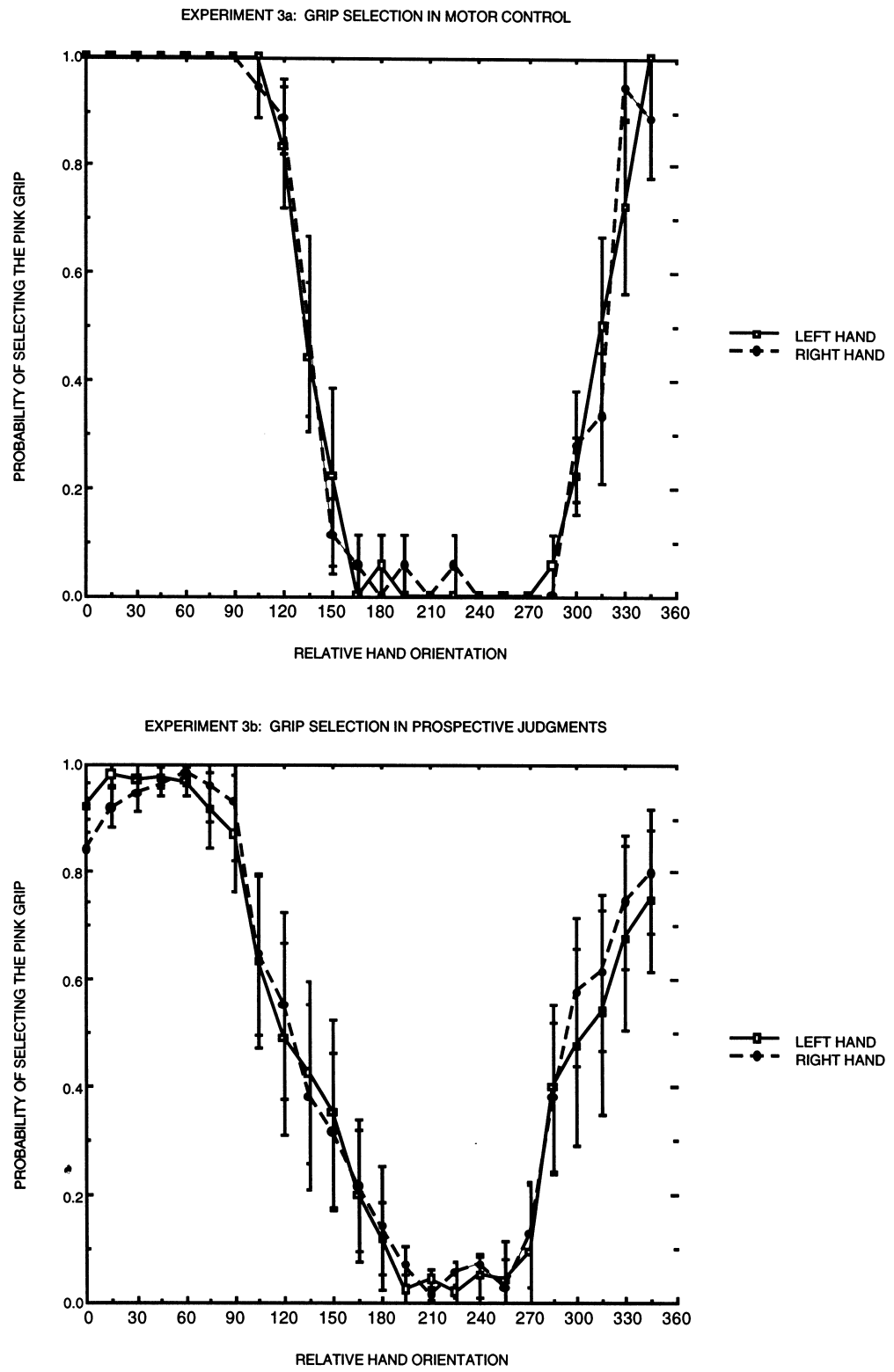

Fig. 6. Relationship between grip preferences and relative hand orientations. Panel A. Because hands obey opposing biomechanical constraints, functions relating grip preferences in $\mathrm{MC}$ to relative hand orientation are nearly identical for the left and right hands. Subjects tend to select grips that minimize perceived awkwardness by avoiding extreme supination or pronation. Panel B. Similar to MC, PJs reflect the biomechanical constraints unique to the two arms. 
critical boundary is reached and they begin to favor the tan grip. This involves supinating the hand $45^{\circ}$, which is also less than the limit of comfortable supination (approx. $100^{\circ}$, Fig. 1), or peak awkwardness $\left(150^{\circ}\right.$ of supination or $225^{\circ}$ expressed as relative hand orientation, Fig. 3, panel A). At $180^{\circ}$ adopting the tan grip involves a neutral posture, identical to the $0^{\circ}$ pink grip. Beginning at $150^{\circ}$ the preferred tan grip involves pronation. At $330^{\circ}$, a grip that would involve $150^{\circ}$ of pronation, subjects again show a preference for the pink grip.

In short, when allowed to select the most natural grip, subjects prefer options that are perceived as least awkward and allow them to remain well within their comfortable ranges of motion (e.g. Rosenbaum et al., 1992). Stated differently, their preferred critical boundaries were consistently less than the absolute critical boundaries (e.g. Cutting, 1982; Jiang \& Mark, 1994; Mark, 1995; Mark et al., 1997).

\subsection{Experiment 3b: prospective judgements}

Similar to Exp. 3a, subjects in the present study were allowed to freely select whether they would prefer an overhand (pronated) or underhand (supinated) grip when engaging a dowel presented in a variety of different orientations. However, as in Exp. 2b, they were required to make these judgements in the absence of any overt movements. That is, they were asked to judge which grips they would prefer if they were to reach for a dowel in different orientations.

Experiment 3a established that subjects preferred grips that minimized awkwardness. Of interest was whether the same would also hold true for PJs. As demonstrated in Exp. 2b, subjects can accurately judge how awkward a particular grip would be to adopt without actually moving. It was reasoned that if mechanisms involved in prospective grip selection are sensitive to the awkwardness of candidate postures, then results should be very similar to those obtained in actual prehension (Exp. 3a). Specifically: (a) subjects should show a preference for grips rated as less awkward (Exp. 2); (b) grip preferences based on the two hands should be virtually identical when expressed as a function of relative hand orientation; (c) transitions from one grip to another should occur before subjects reach their limits of comfortable hand rotation (Exp. 1); and (d) preferred critical boundaries should be highly consistent with those demonstrated in actual prehension (Exp. 3a).

Additionally, if motor imagery is the basis for PJs then the amount of time required to select a grip should increase as a function of both the perceived awkwardness of actually adopting the chosen response (Exp. 2a), and the extent of the movement that would be involved.

\subsubsection{Method}

Six right-handed, University of Massachusetts undergraduates participated in three separate testing sessions lasting approximately $40 \mathrm{~min}$ each for $\$ 15.00$. None of the subjects had participated in any related studies, and all were naive to the hypotheses being investigated. The stimuli and apparatus were identical to those used in Exp. 2b. The procedure was similar to that employed in Exp. 3a, with one important exception: subjects were instructed to choose whether the thumbside of 
their designated response hand would be on the pink or tan end of the dowel if they were to grasp it in the center using a power grip. Subjects were encouraged not to be overly deliberative, and to simply respond in the most natural manner. Again, no mention was made to use imagery when solving this task.

Subjects completed 14 blocks per testing session, each consisting of the stimulus appearing randomly in all 24 orientations (refer to Fig. 1). Hand on which responses were based alternated across blocks. Block order was counterbalanced across subjects.

\subsubsection{Results}

Grip preferences. Grip selection data for each subject were averaged across testing sessions to compute mean probabilities of selecting the pink grip when using the dominant and non-dominant hand at each stimulus orientation.

As expected if grip selection judgements are sensitive to biomechanical constraints, Fig. 6 (panel B) shows that functions relating grip preferences to relative hand orientation for the left and right hands were nearly perfectly correlated, $R=0.99, F(1,22)=1326, P<0.0001, M S e=0.002$. As described above, this reflects the fact that the hands obey joint constraints that are mirror images of one another.

Similar to Exp. 3a, preferred critical boundaries, defined as the orientations at which the probability of selecting one grip exceeded 0.50 , occurred at $135^{\circ}$ and again at $300^{\circ}$ (cf. Figs. 3 and 6 panels B). As with prehension (Exp. 3a), these transitions occurred before subjects would have reached the limits of comfortable pronation or supination estimated in Exp. 1 (Fig. 1), or their critical boundaries (Exp. 2).

Relationship between perceived awkwardness and grip preferences. As anticipated, grip preferences in PJs were inveresely related to perceived awkwardness of imagined prehension (Exp. 2b), $R=-0.96, F(1,46)=552.26, P<0.001$, $M S e=0.01$. Put differently, as with actual prehension, subjects preferred grip options that would minimize awkwardness.

Comparison of Exps $3 a$ and $3 b$. Comparison of panels A and B in Fig. 6 reveals that grip preferences in overt and imagined prehension are strikingly similar, $R=0.94, F(1,46)=336.48, P<0.0001, M S e=0.016$. In both tasks subjects strongly preferred grips rated as less awkward in Exp. 2. Consequently, subjects' preferred critical boundaries tended to be quite similar in both experiments.

Fig. 7 shows differences between PJs and actual grip preferences. Although small in magnitude, the pattern of errors was very similar for each hand, $R=0.89$, $F(1,22)=82.2, P<0.0001, M S e=0.006$. As shown above in Exp. 2b, subjects' PJs of awkwardness were less accurate when they involved grips perceived as highly awkward in prehension (Fig. 4). Similar differences between grip preferences in MC (Exp. 3a) and PJs (Exp. 3b) suggest that prospective grip judgements depend on accurate estimations of awkwardness. Errors tended to increase as a function of perceived awkwardness (Exp. 2a), $R=0.40, F(1,46)=7.6, P=0.008, M S e=$ 0.025 (Fig. 7).

To more closely examine this relationship, the mean probability of selecting the pink grip for each hand at each stimulus orientation was transformed into a decisi- 


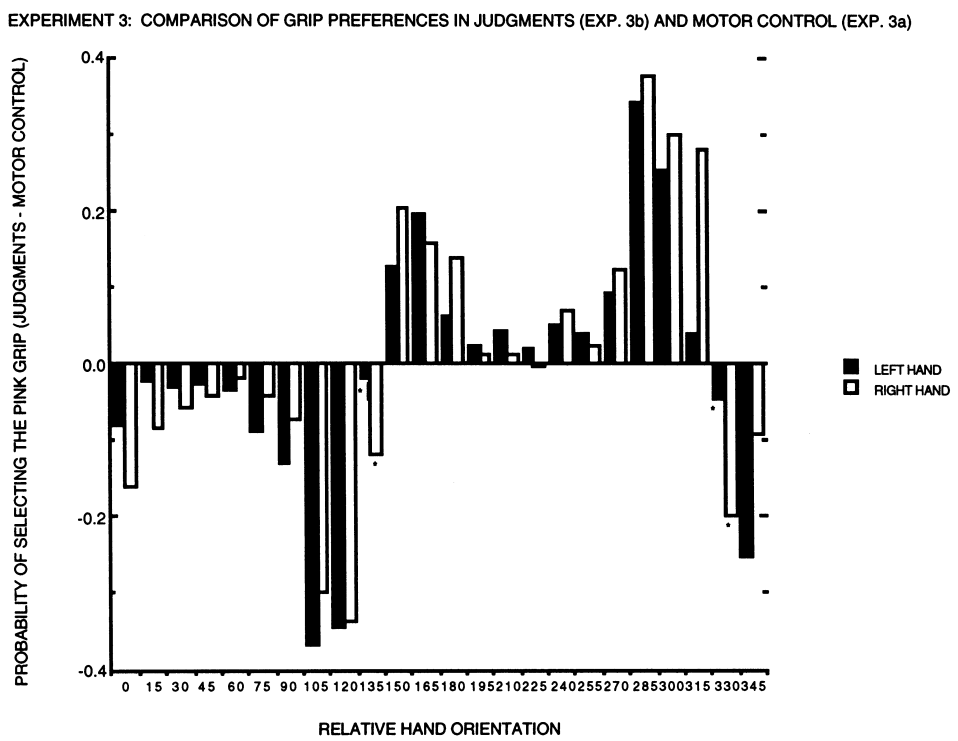

Fig. 7. Differences between grip preferences in MC (Exp. 3a) and PJs (Exp. 3b). Positive values represent overestimates in judgements, and negative values represent underestimates. Asterisks represent preferred critical boundaries between overhand and underhand grips as demonstrated in prehension (Exp. 3a). Note that the tendency for judgements to under- or overestimate actual grip preferences tended to increase and reverse direction near preferred critical boundaries, orientations perceived as being more awkward.

veness index $(D)$ according to the following formula: $D=2$ (probability $\backslash$ of $\backslash$ selecting $\backslash$ the $\backslash$ pink $\backslash$ grip $-\backslash 0.5$ ). The $D$ ranged from $0.0-1.0$ with $D=0.0$ indicating that there was an equal probability of selecting either grip for a particular stimulus orientation, and $D=1.0$ meaning that one grip or the other was always selected with a particular hand when the stimulus was in a given orientation. Because orientations rated as highly awkward clustered around preferred critical boundaries, where neither response option was strongly favored, there was a significant negative relationship between $\mathrm{D}$ and the absolute value of the judgement errors for both the left $(R=-0.57, F(1,22)=10.6, P=0.004, M S e=0.062])$ and for the right hand, $R=-0.71, F(1,22)=22.6, P<0.0001, M S e=0.062$. In other words, PJs tended to be less veridical for those orientations where there was no strong grip preference.

This pattern of errors is also consistent with results of Exp. 2 showing that PJs tend to under- rather than over-estimate grip awkwardness. Consequently, for those orientations where less awkward pronated (i.e. pink) grips were highly preferred in prehension $\left(0-135^{\circ}, 330-345^{\circ}\right)$, there was a tendency for PJs to underestimate the probability of selecting the pink grip. By contrast, for orientations where more awkward supinated (i.e. tan) grips were favored in prehension $\left(150^{\circ}-315^{\circ}\right)$, PJs tended to overestimate the probability of selecting the pink grip.

Response times. Response times greater than $3000 \mathrm{~s}$ and less than $300 \mathrm{~s}$ were eliminated as outliers (approximately 5\% of the total number of responses). Mean 


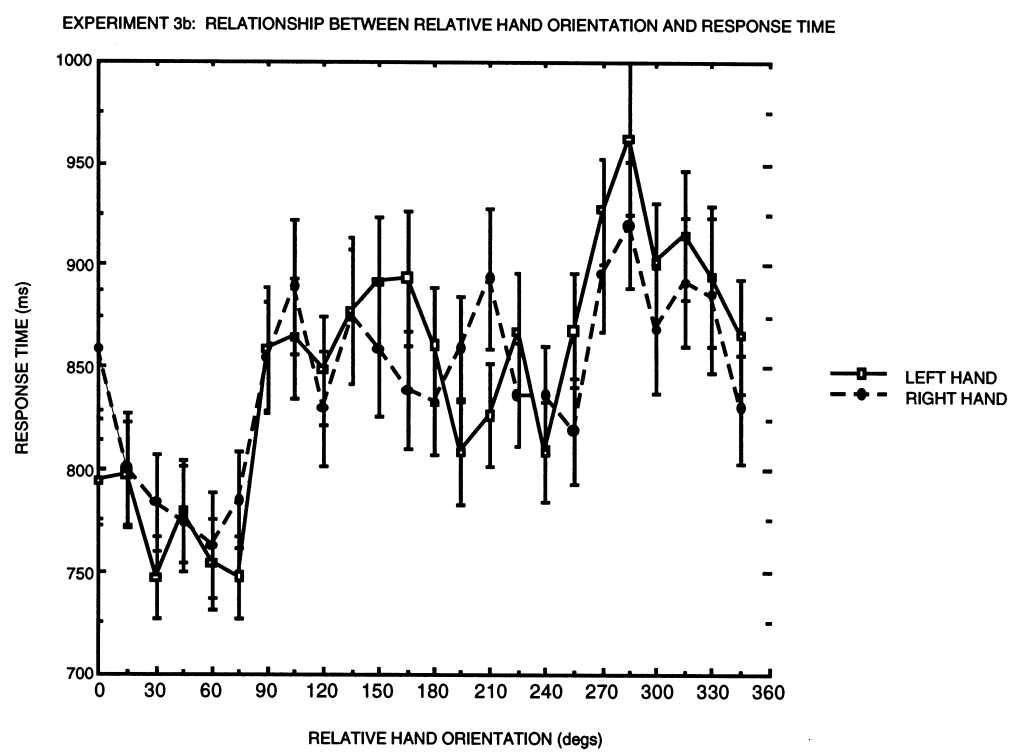

Fig. 8. Relationship between relative hand orientation and response time. Similar to grip preference, times required for movement selection involving comparable rotations of the two hands are well-correlated. As expected if motor imagery is involved in these judgements, this reflects the fact that more awkward grips take longer to select.

RTs were then computed for each subject with each hand at all stimulus orientations. As expected if the timecourse of grip selection was sensitive to their unique biomechanical constraints, Fig. 8 shows that mean RTs for each hand were highly correlated when expressed as a function of relative hand orientation $(R=0.79$, $F(1,22)=37.02, P<0.0001, M S e=1326)$.

Relationship between response time and awkwardness. Consistent with the hypothesis that motor imagery is involved in movement selection, the amount of time required to select a particular grip tended to increase as a function of the chosen grip's rated awkwardness (Exp. 2a), $R=0.344, F(1,46)=6.18, P=0.017, M S e=2288$. Likewise, RTs for decisions based on the left $(R=0.72, F(1,22)=23.4$, $P<0.0001)$ and right hands $(R=0.57, \quad F(1,22)=10.73, \quad P=0.003$, $M S e=1254)$ tended to increase as a function of relative hand orientation.

Relationship between response time and grip preference. There was also a significant negative correlation between $D$ and time required for grip selection $(R=-0.61, F(1,46)=27.0, P<0.001)$, indicating that subjects were generally faster to choose a grip when the stimulus was in an orientation where one option was highly preferred over the other. Conversely, as the probability of selecting either of the two grip options became more equal, RTs tended to increase. As discussed below, this later finding may reflect the need for additional processing when selecting between response options perceived as equally awkward, i.e. as $D$ approaches 0 .

Relationship between response time and extent. The motor imagery hypothesis 
predicts that RTs should increase as a function of the extent of would-be movements. To evaluate this proposal, a variable was created that reflected the number of degrees of angular rotation that would be required to move each hand from the orientation at which its fastest RT was recorded $\left(30^{\circ}\right.$ for the left hand and $45^{\circ}$ for the right hand), into the orientation of the stimulus using the biomechanically possible path. Note that these starting orientations are approximately the same orientations as those in which subjects' hands were actually positioned (i.e. $90^{\circ}$; see Parsons, 1994 for a comprehensive discussion of this issue). In creating this variable, grip preference data were used to determine the preferred direction (pronation or supination) of rotation for each hand. Whichever grip was selected better than $50 \%$ of the time was taken as the preferred grip. This strategy provides conservative estimates of the relationship between RTs and angular distance because it makes no distinction between orientations where there were weak versus strong grip preferences. Nevertheless, as shown in Fig. 9, there was a highly significant relationship between RT and the angular distance variable for both the left $(R(1,22)=17.3$, $P<0.0001, M S e=1.93)$ and right hands, $R=0.49, F(1,22)=6.8, P=0.016$, $M S e=3.4$. The best-fitting linear function relating RT to angular distance had a slope of 0.989 , suggesting that the rate of this mental transformation was approximately $989^{\circ} / \mathrm{s}$.

\subsubsection{Discussion}

Experiment 3 revealed that, when allowed to freely choose between two response

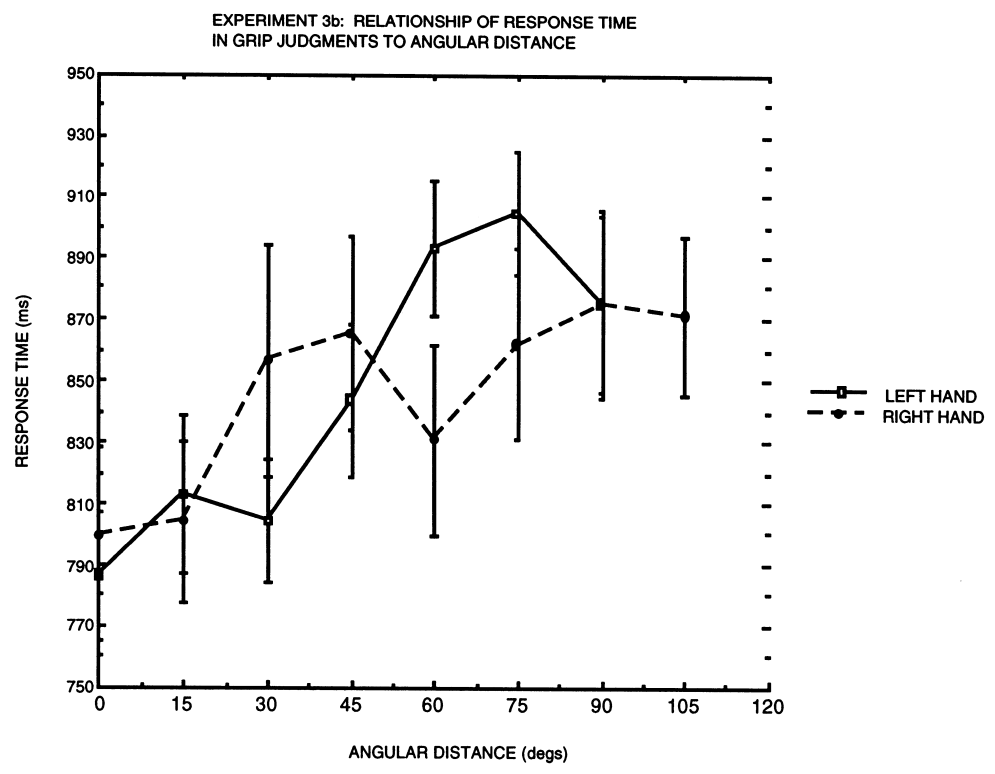

Fig. 9. Relationship between response time and angular distance. Response times for PJs of grip tend to increase as a function of the angular distance between each hand's fastest orientation, approximately the actual position of subjects' hands, and the orientation of the stimulus. 
options, subjects' prospective action judgements are remarkably veridical; i.e. changes in the manipulandum's orientation had virtually identical effects on grip selection in both prehension (Exp. 3a) and judgements (Exp. 3b). This differs from results of several previous studies in which subjects were required to estimate their absolute critical boundaries for a prespecified action (e.g. Carello et al., 1989; Heft, 1993; Jiang \& Mark, 1994; Mark, 1995; Mark et al., 1997, Exp. 1; Robinovitch \& Cronin, 1997). In both tasks, subjects strongly favored grips that were rated as less awkward in Exp. 2, and as a result preferred critical boundaries in both tasks were well within the range of comfortable hand rotation established in Exp. 1.

Similar to Exp. 2, differences between grip preferences in MC and PJ conditions were highly systematic, with judgements being less accurate for those orientations that would have involved more awkward movements. Because judgements at these orientations also took longer to make, this pattern is not attributable to a speedaccuracy trade-off. Instead, this is what would be expected if PJs involved imagining, or mentally-simulating, reaches.

Response times are also consistent with the use of motor imagery. As would be true for actual movements, grips that would have required adopting more awkward postures (Exp. 2a), took longer to select. And, when expressed as a function of relative hand orientation, RTs for judgements based on the left and right hands were very similar (Fig. 8), suggesting that speed was influenced by the inversely related biomechanical constraints of the two hands. Indeed, closer inspection revealed that RTs tended to increase as a function of the extent of hand rotation moving in the biomechanically possible direction (Fig. 9).

It is important to realize that these results cannot be attributed to simple mental rotation, which seems to follow the shortest path (e.g. Shepard \& Cooper, 1982). If this were the case, then one would not expect to find different effects of stimulus orientation on judgements based on the left and right hands. The nonlinear relationships between RTs and stimulus orientations depicted in Fig. 8 clearly indicate that this is not the case. Instead, these transformations appear to be rapid simulations of movements in biomechanically plausible trajectories (Fig. 9). The average rate of these transformations $\left(989^{\circ} / \mathrm{s}\right)$ is considerably faster than typically observed for mental rotation of three-dimensional objects (e.g. Shepard \& Cooper, 1982), which tends to fall within the range of approximately 40- 400\% (Tarr, 1995). However, it is similar to the estimated rate of $886 \%$ for prospective awkwardness judgements in Exp. $2 b$.

Finally, subjects were generally faster to choose a grip when the stimulus was in an orientation where one option was highly preferred over the other, i.e. $D$ approaching 1.0. This relationship may reflect a more difficult comparison when simulations of both grip options yield similar awkwardness ratings. This possibility will be developed further in Section 6 below.

\section{Experiment 4: hand selection}

In the previous experiments, subjects were instructed to use a particular hand 
when responding. However, an important aspect of planning prehensile movements involves selecting which hand to use. The fourth experiment was designed to compare the effects of changes in stimulus orientation on hand selection in both $\mathrm{MC}$ and PJ conditions. It was reasoned that if hand selection is based on an evaluation of awkwardness, then PJs (Exp. 4b) should again be very similar to actual hand preferences (Exp. 4a).

As in Exp. 2, subjects were asked to grasp the dowel so that the thumbside of their hand was always toward the pink or tan end. However, they were now free to select either their dominant (right) or non-dominant (left) hands. Because these subjects were right-handed, a bias toward using the right hand was anticipated. Nevertheless, if hand selection is based on an evaluation of awkwardness, then a preference for the non-dominant left hand should occur at orientations where use of the right hand would be more cumbersome.

This experiment also provided an additional opportunity to evaluate the hypothesis that PJs involve motor imagery. Implicit in the motor imagery hypothesis is the assumption that simulated movements are not task-specific: Because they presumably involve imagining entire movements, as constrained by the demands of the task, the information available in these simulations should be useful for solving many different aspects of the movement selection problem. If so, then, as observed in Exps. $2 b$ and 3b, RTs in hand selection judgements should increase as a function of both the awkwardness and extent of the would-be movement.

\subsection{Experiment 4a: motor control}

\subsubsection{Method}

Six right-handed undergraduates participated in a single 30 min testing session for $\$ 5.00$. None of the subjects participated in any related studies, and all were naive to the hypotheses being investigated. The stimulus and apparatus were identical to those described in Exp. 1. The procedure was similar to that described in Exp. 2a. Subjects were instructed to grasp the dowel in the center using a power grip such that the thumbside of their hand was always toward the designated end. On alternating blocks the designated end was either pink or tan. They were, however, free to use either their left or right hands, depending on which was most comfortable. It was emphasized that subjects should not make their response until the experimenter instructed them to reach. Subjects completed 6 blocks of trials, each consisting of 24 stimulus orientations presented in random order (Fig. 2).

\subsubsection{Results}

The probability of selecting the dominant right hand was calculated separately for each subject at every stimulus orientation. In Fig. 10 (panel A) mean probability values are plotted as a function of relative hand orientation for the right hand (see Fig. 1). Similar to Exps. 2 and 3, data from identical stimulus orientations in the pink and tan blocks have been combined and are displayed as though subjects were instructed to place their thumbs on the pink end. As expected, subjects tended to select the hand that allowed them to adopt grips that were perceived as being less 
EXPERIMENT 4a: HAND SELECTION IN MOTOR CONTROL

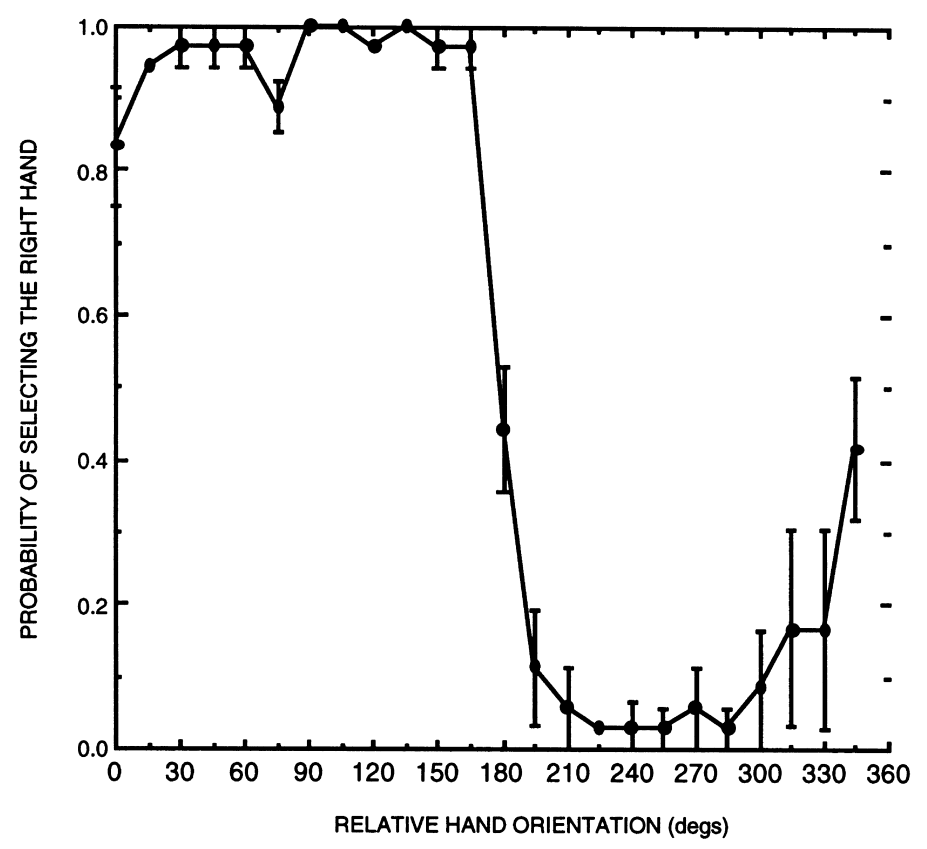

EXPERIMENT 4b: HAND SELECTION IN PROSPECTIVE JUDGMENTS

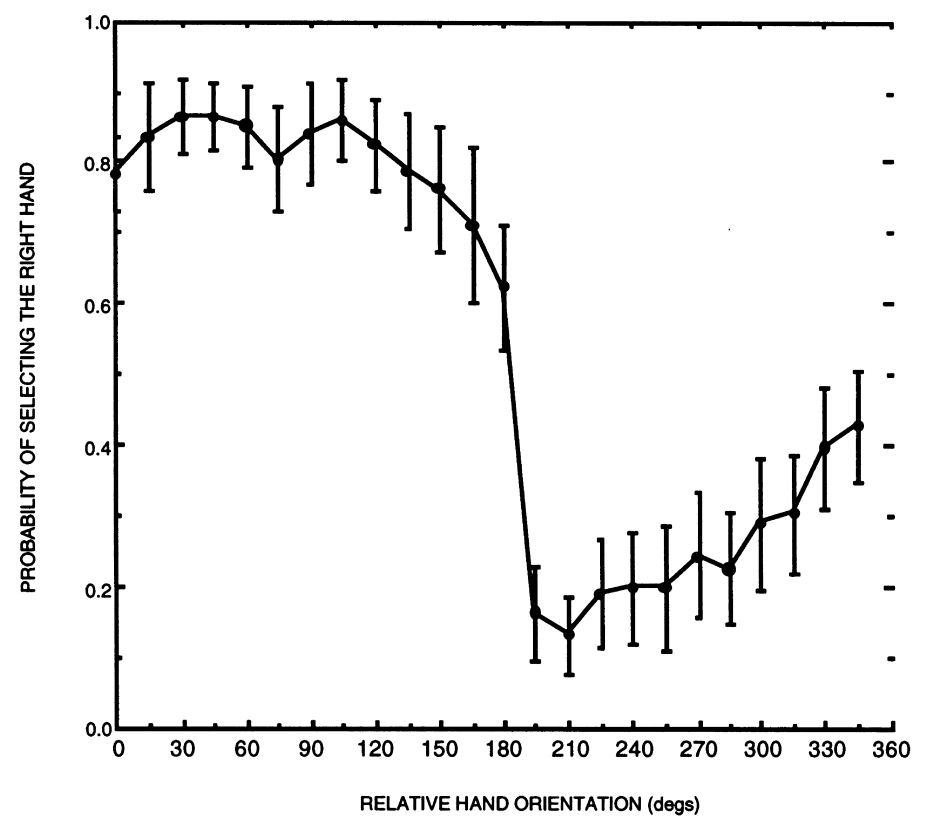


awkward. Consequently, there was a negative correlation between the probability of selecting the dominant right hand, and the rated awkwardness of right hand grips (Exp. 2), $R=-0.65, F(1,21)=15.65, P<0.001, M S e=0.139$. Subjects showed a strong preference for the dominant right hand when the stimulus was oriented vertically (with the pink end pointed upward), an orientation perceived as equally comfortable with both hands (Fig. 3, panel A). The right hand was favored until $180^{\circ}$, at which point a preferred critical boundary was reached and subjects began to favor the left hand. The fact that the transition between preferred hands occurred at $180^{\circ}$ indicates that subjects were not biased to select their dominant right hand. In fact, subjects chose to switch to a pronated grip with their left hand in order to avoid supinating the right hand by as little as $15^{\circ}$, as would be required for the $345^{\circ}$ stimulus. This occurred even though the comfortable limit of right hand supination is approx. $100^{\circ}$ (Fig. 1).

It is noteworthy that preferred critical boundaries in hand preference occurred at approximately the same orientations where subjects in Exp. 3a switched from underhand (supinated) and overhand (pronated) grips with the right hand (cf. Fig. 6, panel A and Fig. 10, panel A). Because subjects in the present experiment were not free to change grips, they instead switched hands in order to avoid adopting highly awkward postures.

\subsection{Experiment $4 b$ : prospective judgements}

As with grip selection, the previous experiment demonstrated that subjects tend to respond with the hand that allows them to avoid adopting awkward postures. Would the same be true for PJs of hand preference? To the extent that these judgements are veridical, hand preferences in the present task were expected to be consistent with those observed in Exp. 4a. More precisely, it was predicted that subjects would prefer the non-dominant hand for those stimulus orientations that would require pronating the dominant hand by more than $180^{\circ}$. At all other orientations there would be preference for the dominant right hand.

In Exp. 3b, it was argued that prospective grip judgements are based on use of motor imagery to evaluate the awkwardness of potential response options. If hand judgements also involve simulated movements, then RTs should increase as a function of awkwardness of the selected grip. Likewise, increases in RT are also

Fig. 10. Relationship between hand preferences and relative hand orientations. Panel A. As in grip selection (Exp. 3), subjects in the MC condition select the hand that allows for the least awkward posture. Note that results are plotted as a function of relative orientation of the right hand (Fig. 2). The nondominant left hand is preferred for orientations that would involve grips requiring supination of the dominant hand, or pronation of the dominant hand by $180^{\circ}$ or more. The dominant hand is preferred for all other orientations. However, there is a bias in favor of selecting the dominant right hand when either response would equally awkward/comfortable (e.g. $0^{\circ}$ ). Panel B. As in MC (Exp. 4a), PJs reflect a strong tendency to prefer the hand that allows the least awkward grip. Likewise, there is a slight bias toward selecting the dominant right hand, at orientations perceived as equally awkward/comfortable(e.g. $\left.0^{\circ}\right)$. However, the non-dominant left hand is preferred for stimulus orientations requiring supination of the dominant hand, or pronation by $180^{\circ}$ or more. The dominant hand is preferred for all other orientations. 
expected as the angular distance between the final grip and the approximate resting position of subjects' hands increases. If results of these simulations are used to select a response hand, then judgements should also be fastest when one option is clearly less cumbersome than the other, and slower when both responses would involve adopting postures perceived as comparably awkward (Exp. 2a).

Response times for hand judgements might also shed light on the sequence of processes involved in these simulations. For instance, if subjects initially plan with their dominant hand, switching to the non-dominant hand only after determining that substantial awkwardness would be involved, then RTs for the right hand should always be faster than for the left. However, if subjects simulate the actions of both hands simultaneously, then no such difference would be anticipated.

\subsubsection{Method}

Twelve, right-handed undergraduates participated in a single 40 min testing session for course credit. None of the subjects were involved in any related experiments, and all were naive to the hypotheses being evaluated. The study employed the same stimuli, and apparatus as used in Exps. $2 \mathrm{~b}$ and $3 \mathrm{~b}$, but the procedure differed in several respects. The study was again divided into 14 blocks of trials each consisting of the dowel appearing in 24 orientations in random order (Fig. 2). As in Exp. 4a, subjects were instructed to determine which hand would be used if they were to reach for the dowel such that the thumbside of their hand would be toward the designated end of the dowel. Blocks in which the designated end was pink or tan were presented in alternating order, and the sequence of blocks was counterbalanced across subjects. The computer measured the time from the onset of the stimulus until subjects responded by saying " left" or " right" into the microphone, and responses were manually entered into the computer after each trial by the experimenter.

\subsubsection{Results}

As described for Exp. 4a, the mean probability of selecting the dominant right hand was computed separately for each subject at each stimulus orientation.

Relationship between hand preferences and awkwardness. As predicted, there was a negative correlation between perceived awkwardness ratings with the right hand (Exp. 2a), and the probability of selecting the right hand in the present task, $R=-0.708, F(1,22)=22.256, P<0.001, M S e=0.044$. Similar to MC, subjects avoided supinated grips, instead preferring to use a less awkward pronated grip with the non-dominant left hand even for those orientations that were well within the bounds of comfortable right hand supination, i.e. $270^{\circ}-345^{\circ}$.

Comparison of Exps. $4 a$ and $4 b$. As a consequence of preferring less awkward postures, PJs were very similar to those observed in MC (Exp. 4a), $R=0.98$, $\mathrm{F}(1,22)=501.3, P<0.0001, M S e=0.004$ (cf. Fig. 10, panels A and B). Similar to grip selection, differences in hand preferences between $\mathrm{MC}$ and $\mathrm{PJ}$ tasks tended to increase at those orientations perceived as being more awkward with either the left $(R=0.63, F(1,22)=14.7, P=0.001, M S e=0.73)$, or right hand $(R=0.46$, $F(1,22)=6.0, P=0.023, M S e=1.07)$. As shown in Fig. 11 , this had the effect of causing subjects to make conservative judgements that underestimated the prob- 


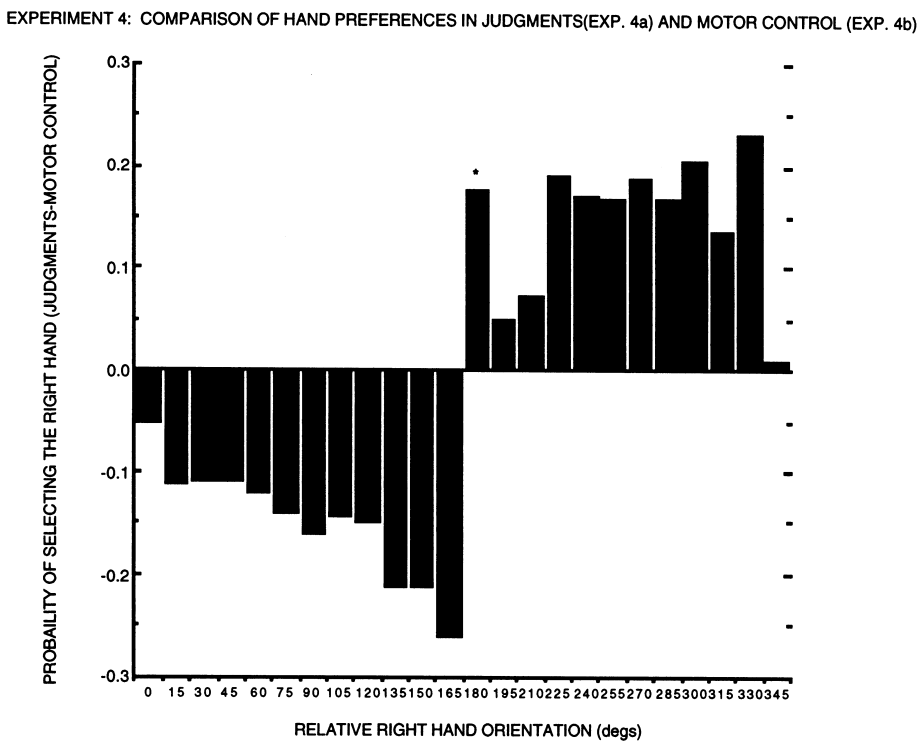

Fig. 11. Differences between hand preferences in MC (Exp. 4a) and PJs (Exp. 4b). Positive values represent overestimates in PJs versus MC, and negative values represent underestimates. Asterisks represents the preferred critical boundary between right and left hand preferences as demonstrated in prehension (Exp. 4a). Although judgements were highly veridical, there was a conservative tendency to overestimate the probability of selecting the right hand at those orientations where the left hand was actually preferred, and to underestimate the probability of using the right hand at those orientations where it was the favored response.

ability of selecting the right hand for those orientations where the right hand actually dominated $\left(0^{\circ}-165^{\circ}\right)$, and overestimated the probability of using the right hand at those orientations where the left hand was actually preferred $\left(180^{\circ}-345^{\circ}\right)$. Consequently, there was a highly significant relationship between the probability of selecting the right hand in PJs (Exp. 4b), and differences between the probability of selecting the right hand in judgements (Exp. 4b) versus MC (Exp. 4a), $R=0.83$, $F(1,22)=52, P<0.0001, M S e=0.026$.

Response times. Approximately $6 \%$ of trials had RTs greater than $3000 \mathrm{~ms}$ or less than $300 \mathrm{~ms}$. These were considered outliers and eliminated prior to analysis. Mean RTs were then calculated for each subject and each stimulus orientation. There was no RT advantage for trials on which the dominant hand was selected, $F(1,11)=1.438, P=0.256$. This is consistent with a model in which subjects evaluate reaches with both their dominant and non-dominant hands prior to making their selection.

Relationship between response time and perceived awkwardness. As would be expected if subjects were basing their judgements on simulated movements, there was a negative correlation between the time required to select a response hand and perceived awkwardness ratings obtained in Exp. 2a, $R=-0.60, F(1,22)=12.3$, $P=0.002, M S e=3172$. 


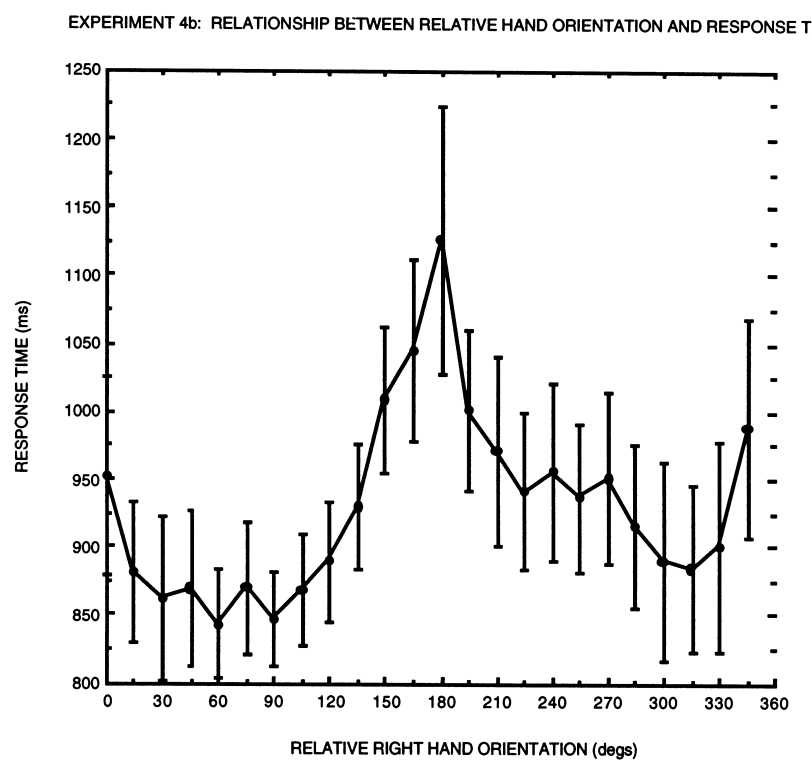

Fig. 12. Relationship between relative hand orientation and response time. Response latencies for prospective hand judgements tend to increase around preferred critical boundaries; orientations that are also perceived as being more awkward. Consistent with grip selection (Exp. 3b), the fastest RT for orientations where the right hand was preferred occurred at $30^{\circ}$, while the fastest orientation where the left hand was preferred was $45^{\circ}$. Both orientations would involve slightly pronated grips.

Relationship between response time and hand preference. Fig. 12 shows that RTs peaked at $180^{\circ}$, the preferred critical boundary at which subjects made the transition between right and left hand preferences (Fig. 10, panel B). Subjects' hand preferences for orientations around this critical boundary tend to be less consistent (Fig. $10 \mathrm{~b}$ ), suggesting that RT may be inversely related to decisiveness. To explore this possibility, mean hand preferences for each stimulus orientation were transformed to yield an estimate of decisiveness $(D)$ according to the following formula: $D=2$ (probability of selecting the right hand-0.5). As in Exp. 3b, values ranged from $0-1.0$, with $D=0.0$ meaning that there was an equal probability of selecting either hand for a particular stimulus orientation, and $D=1.0$ meaning that one hand or the other was always selected for a given stimulus orientation. The $D$ variable was then regressed onto mean RTs. Similar to grip selection (Exp. 3b), RTs tended to be shorter for orientations where one hand was highly preferred over the other, and slower for those orientations where subjects did not demonstrate a strong hand preference, $R=0.49, F(1,22)=6.9, P<0.016, M S e=3770$. This is consistent with a model in which hand selection is based on a comparison of the perceived awkwardness of both response options. As developed in Section 6 below, additional time may be required to select a response when simulations of the options yield similar results. 
Relationship of response time and extent. Like PJs of grip selection (Exp. 3b), the fastest RTs occurred at relative hand orientations that would involve adopting moderately pronated postures: $45^{\circ}$ for those stimuli where the left hand was preferred, and $30^{\circ}$ for those stimuli where the right hand was preferred (Fig. 2). These are similar to the actual positions of subjects hands (i.e. $90^{\circ}$ ). As predicted by the motor imagery hypothesis, RTs tended to increase as a function of the angular distance between the chosen response hand and these fastest orientations using the biomechanically possible path. Fig. 13 shows that this was true for stimulus orientations where either the left $(R=0.80, F(1,9)=12.23, P=0.007, M S e=707)$ or right hands $(R=0.92, \quad F(1,11)=63.9, \quad P<0.0001, \quad M S e=1229.8)$ were preferred. The slope of the best-fitting linear function relating RTs to angular distance suggests that the rate of these transformations averages $1512 \%$ s. As in grip selection, this is considerably faster than transformations in mental rotation studies involving 3D objects (e.g. Shepard \& Cooper, 1982; Tarr, 1995). Similar to Exps. $2 b$ and $3 b$, this pattern is consistent with the hypothesis that movement selection involves a rapid, analog simulation of response options.

\subsubsection{Discussion}

As with grip selection, when allowed to freely choose between two response

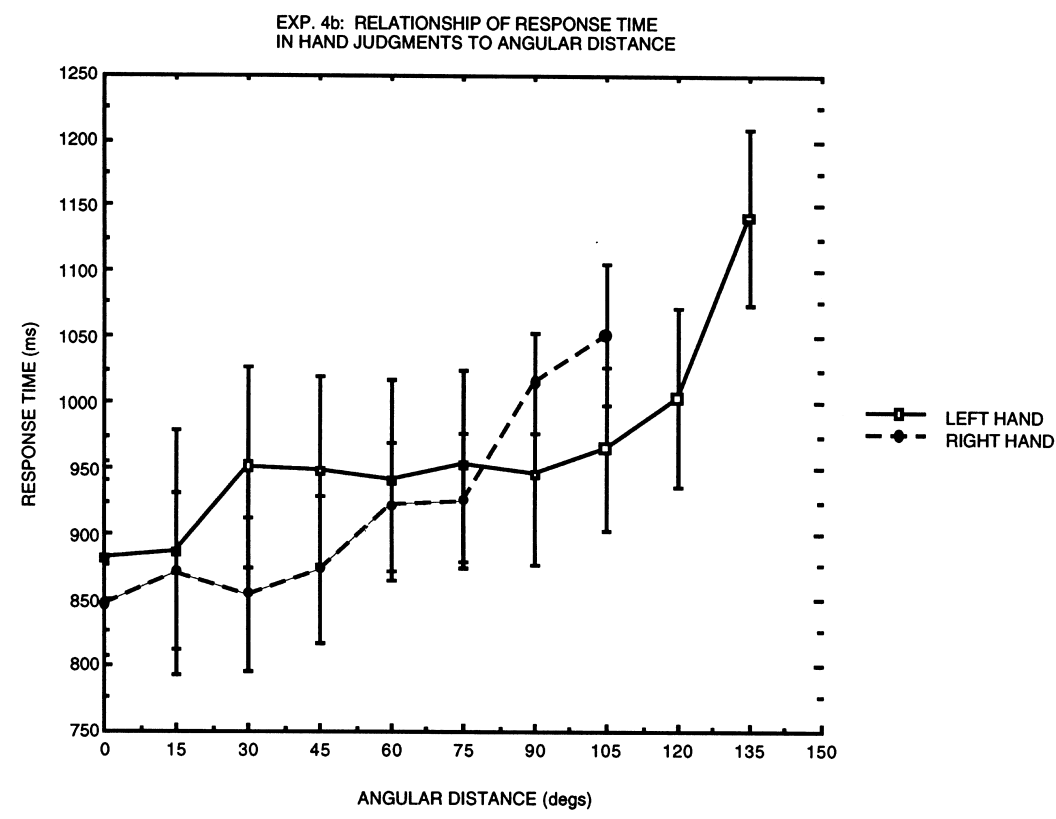

Fig. 13. Relationship between response time and angular distance. Response times for prospective hand judgements tend to increase as a function of the angular distance between the fastest orientation at which the left $\left(45^{\circ}\right)$ or right $\left(30^{\circ}\right)$ hand was preferred, and the number of degrees required to rotate that preferred hand into the orientation of the stimulus following the biomechanically possible path. 
options, subjects' PJs are highly veridical. This contrasts with earlier findings indicating that subjects tend to incorrectly estimate limitations of their ability to perform a single action (e.g. Carello et al., 1989; Heft, 1993; Jiang \& Mark, 1994; Mark et al., 1997, Exp. 1; Robinovitch \& Cronin, 1997). In both MC (Exp. 4a), and PJ tasks (Exp. 4b), subjects tended to select the hand that would allow the least awkward grip, even when this meant going to the non-dominant hand. As a consequence, these right-handed subjects showed a minimal bias toward selecting the dominant hand for this task. In fact it occurred only for $0^{\circ}$ stimuli. Even for orientations that were well within the limits of comfortable supination with the right hand, i.e. $270^{\circ}-345^{\circ}$, subjects preferred to adopt pronated grips with the non-dominant left; grips that were perceived as being less awkward (Exp. 2). It seems likely that a bias favoring the dominant hand might emerge in both MC and PJs if the tasks demanded finer coordination, e.g. rotating the dowel to a very precise orientation. This possibility is currently under investigation.

As anticipated if awkward postures are more difficult to simulate accurately (e.g. Parsons, 1994), PJs were less accurate when they involved grips perceived as being highly awkward (Exp. 2a). Likewise, RTs increased as a function of perceived awkwardness of the would-be movement (Exp. 2a). Like awkwardness (Exp. 2b) and grip selection (Exp. 3b), the time required for PJs of hand selection also increased as a function of the extent of the would-be movement. The rate of these transformations again seemed to be considerably faster $\left(1500^{\circ} / \mathrm{s}\right)$ than is typical of rotation of visual mental images (e.g. Shepard and Cooper, 1982; Tarr, 1995), and followed the biomechanically possible trajectory rather than the shortest path. This can be considered further support for the motor imagery hypothesis.

As in Exp. 3b, shortest RTs were observed for stimuli in orientations similar to those in which subjects' hands were actually positioned, suggesting that PJs may involve mental transformations of one's own hands from their present locations into the postures demanded by the stimulus (Parsons, 1994). Additionally, the fact that there was no difference in the amount of time required for selection of the left versus right hand suggests that both alternatives were evaluated before movement selection. In other words, subjects did not plan with their dominant hand by default, only switching to the non-dominant if this option appeared too cumbersome. Additional research is required to determine whether planning for both hands is accomplished in a serial or parallel fashion.

In short, results are consistent with those of grip selection in suggesting that PJs are both highly accurate, and involve motor imagery. For the majority of postures these simulations tend to be veridical, yielding results consistent with actual prehension. However, for those stimulus orientations that would involve adopting awkward postures, simulations are both less accurate and slower. These findings therefore concur with a number of previous studies showing that difficult actions require more time to image (e.g. Decety \& Jeannerod, 1995; Decety, Jeannerod, \& Prablanc, 1989; Georgopoulos \& Massey, 1987; Parsons, 1987b), and with Parson's (1994) observation that awkward actions are harder to simulate. 


\section{General discussion}

\subsection{Accuracy of prospective judgements}

The studies detailed above were intended to address two questions concerning PJs about actions. First, when subjects are allowed to choose the response that seems most appropriate for a given prehension task, how similar are their PJs to their actions? The answer, for both grip and hand selection, is that PJs are extremely veridical. This result contrasts substantially with several previous studies suggesting that PJs often underestimate subjects' actual abilities (e.g. Carello et al., 1989; Heft, 1993; Jiang \& Mark, 1994; Mark, 1995; Mark \& Vogele, 1988; Mark et al., 1997, Exp. 1). One possibility is that these earlier observations simply reflect subjects' relative lack of experience performing actions at their physical limits, or absolute critical boundaries (cf. Mark et al., 1997, p. 1376). When given the opportunity to choose, subjects typically avoid such awkward postures, instead switching to a more comfortable alternative (see also Jiang \& Mark, 1994; Mark, 1995; Mark et al., 1997).

Second, what mechanisms are used in making judgements about would-be actions? While previous studies have couched prospective action judgements in the language of directly perceiving objects' affordances (e.g. Mark et al., 1997), the presents results suggest that subjects base their responses on analog simulations of would-be actions, or motor imagery (e.g. Jeannerod, 1994; Johnson, 1998; Kohl \& Fisicaro, 1996). Several aspects of the present findings support this motor imagery hypothesis. Throughout these experiments decisions that would have involved adopting awkward postures consistently required more time to make, and were less similar to actual movements, i.e. less accurate. One possibility is that subjects based their movement selections on stored motor memories. Because awkward postures tend to be avoided they may be less richly represented in memory, and consequently lead to slower and less accurate judgements. However, this hypothesis does not explain why time required to make these judgements tended to increase as a function of the angular distance between the approximate locations of the subjects' hands and the orientation of the chosen posture through the biomechanically defined trajectory. A linear increase in RT with angular distance is expected if PJs involve on-line, analog, simulations of movements. From this perspective awkward postures are not only more difficult to physically adopt, but also to mentally simulate. As suggested by Parsons (1994), this may be attributable to the involvement of complicated, multi-joint simulations that place greater demands on the representational system. Rates of simulated actions in the present studies appeared to be considerably faster than what has been observed in studies of mental rotation involving 3D objects (e.g. Shepard \& Cooper, 1982; Tarr, 1995). However, the present rates are similar to rapid transformations estimated in the recognition of disoriented letters (Jolicoeur \& Landau, 1984), and two-dimensional, symmetrical, shapes (Tarr \& Pinker, 1990; Johnson, 1991). In this limited sense the present findings are consistent with evidence indicating use of common mechanisms in motor planning and mental rotation (e.g. Pellizzer and Georgopoulos, 1993; Wexler, Kosslyn \& Berthoz, 
1998). Nevertheless, representations involved in the present studies would seem to differ substantially from those involved in classic mental rotation. Instead of following the shortest path, these transformations follow the path dictated by biomechanical constraints on joint rotation, suggesting the involvement of somatomotor, as opposed to visual, representations. Consequently, these findings also have potentially important implications for understanding the relationship between motor imagery and planning.

\subsection{Relationship between motor imagery and motor planning}

There is a growing body of evidence suggesting that motor imagery involves a subsystem of those mechanisms involved in motor planning and/or control (e.g. Crammond, 1997; Jeannerod, 1994; 1995; Johnson, 1998; Johnson et al., 1999; Stephan et al., 1995). However, precisely which components are common to both motor imagery and planning is not presently known. On the one hand, beginning with the work of Roland, Skinhoj, Lassen \& Larsen, (1980), two decades of functional neuroimaging studies have demonstrated that imagining movements activates areas involved in motor planning and control including: cerebellum and basal ganglia (e.g. Decety, Philippon \& Ingvar, 1988; Parsons et al., 1995), supplementary and pre-motor areas (e.g. Decety et al., 1994; Deiber, Ibanez, Honda, Sadato, Raman \& Hallett, 1997; Parsons et al., 1995; Rao et al., 1993), posterior parietal cortex (e.g. Stephan et al., 1995) and in some cases even primary motor and somatosensory cortex (e.g. Hallett, Fieldman, Cohen, Sadato \& Pascual-Leone, 1994; Pfurtscheller \& Neuper, 1997; Roth et al., 1996). Studies of brain-injured patients suggest that these areas may contribute to different components of motor imagery. Patients with lesions of posterior parietal cortex, for example, often have difficulty generating accurate motor images (e.g. Sirigu, Duhamel, Cohen, Pillon, Dubois \& Agid, 1996), while patients with primary motor cortex lesions may be capable of generating motor images, but show considerable slowing when imagining movements of afflicted muscles (e.g. Sirigu, Cohen, Duhamel, Pillon, Dubois, Agid \& PierrotDeseilligny, 1995). Likewise, Parkinson's patients also may show comparable slowing of actual and imagined movements (Dominey, Decety, Broussolle, Chazot \& Jeannerod, 1995). On the other hand, motor imagery is dissociable from motor control (Johnson, 1999; Johnson et al., 1999). Apart from the obvious fact that imagery does not involve overt movements, paralyzed individuals may retain the ability to generate motor images and internally simulate movements (e.g. Decety \& Boisson, 1990; Gandevia, 1982; Hildebrandt \& Zeiger, 1995; Johnson, 1999; Weiss, Hansen, Rost \& Beyer, 1994).

One popular interpretation of the similarities between motor imagery and action is that imagery is the conscious experience of a normally non-conscious premotor plan (Jeannerod, 1994, 1995). From this perspective motor imagery depends on the existence of a computed premotor plan; when execution of the plan is inhibited, motor imagery is experienced. The present studies, however, suggest that motor imagery may actually contribute to solving the problem of movement selection, a major component of constructing the premotor plan (e.g. Bernstein, 1967; Rosen- 
baum, 1991). This alternative imagery as planning hypothesis states that response selection may involve mentally transforming somatomotor representations of the effectors in order to anticipate the consequences of upcoming actions in advance of their execution. In particular, motor imagery may be involved when selecting from among a discrete number of candidate response options. Imagery might therefore be used after other processes have narrowed the number of potential response options, or when only a small number of solutions exist, as was the case in the present tasks.

Primary motor cortex may play an important role in premovement simulations. Georgopoulos and colleagues (e.g. Georgopoulos, Lurito, Petrides, Schwartz \& Massey, 1989; Lurito, Georgakopoulos \& Georgopoulos, 1991) have shown that during movement preparation there is a systematic transformation of the directional sensitivities of large populations of cells into the plane of the upcoming reach. Response times from a comparable task suggest that similar pre-motor transformations may occur in humans as well (Georgopoulos \& Massey, 1987).

\subsection{Prospective action model (PAM)}

While additional work is needed to determine the role of analog transformations in planning actual movements, the present findings do suggest a provisional model of processes involved in PJs. According to the PAM, judgements about how one is likely to act involve several stages of processing. Initially, subjects encode the orientation of the target stimulus, activate somatomotor representations of their hand/arms, and transform these into the orientations required by each response option in a manner respectful of prevailing biomechanical constraints. It is unclear from the present data whether these simulations occur serially or in parallel. However, the fact that there were no differences between RTs for the left and right hands in either grip (Exp. 3) or hand (Exp. 4) selection suggests that both options are simulated before a decision is made (see also Parsons, 1989b, 1994). In other words, there is no default preference for, say, an overhand or right handed response. Response time profiles for both grip and hand selection suggest that these transformations begin either at the current location of subjects' hand(s) (e.g. Parsons, 1994), or from slightly pronated positions. These transformations appear to be analog, but unlike visual mental rotation simulated movements appear to be very rapid, on the order of $1000 \%$, and to respect biomechanical constraints of the involved effectors.

According to the PAM, results of these simulations are estimates of the biomechanical demands, or awkwardness, associated with each of the respective response options. Further, these estimates tend to be most accurate when they concern less awkward movements. Awkwardness values are then submitted to a primary comparator. If levels of awkwardness associated with the response options differ substantially, then the least awkward posture is immediately selected. If, however, simulations of two or more response options yield similar levels of awkwardness, then an additional decision stage is necessitated, and comparatively more processing time will be required. This is reflected in the fact that stimulus orientations for which neither option was highly preferred had longer RTs. 
Further work is underway to determine whether the PAM can be extended to PJs involving other modes of action, and its ability to accommodate specific motor planning phenomena.

\subsection{Conclusions}

Motor imagery is presently undergoing a surge of interest similar to the study of visual imagery in the 1970s and 1980s (e.g. Annett, 1995; Jeannerod, 1994). As with visual imagery, claims regarding the involvement of motor imagery in problem solving will have to be staked on empirically rigorous grounds. Toward this end, researchers in the area of motor imagery can benefit from the history of the so-called " imagery debate"' (see Kosslyn, 1980), and in doing so perhaps avoid many of the pitfalls that beset early visual imagery research (for a comprehensive review and analysis see Kosslyn, 1995). The following criteria are offered as an initial step in this direction.

Avoidance of demand characteristics. Studies of motor imagery phenomena should employ tasks that are designed to minimize demand characteristics. That is, phenomena should be quantitatively measured using tasks that are predicted to require imagery to solve, rather than instructing subjects to explicitly imagine making particular movements and relying on their introspective reports. In the present tasks care was taken to avoid encouraging subjects to " use imagery" or " imagine moving their hands" . Put differently, these tasks required implicit motor imagery (Parsons et al., 1995). In debriefing sessions subjects did not report visualizing their hands reaching out for the stimulus objects.

Compatibility with results of movement control tasks. Performances on tasks predicted to involve motor imagery should be quantitatively similar to results from control tasks that involve comparable movements. This comparison can be used to estimate the extent to which representations involved in motor imagery make explicit constraints on motor control. For instance, statistical comparisons of MC and PJ tasks in Exps. 2- 4 consistently revealed highly similar patterns, suggesting that imagined movements obeyed biomechanical constraints on hand rotation.

Reliance on imagery not memory. In order to avoid reliance on memory of how a specific action was performed, as opposed to the results of an on-line mental simulation, care should be taken to avoid practising subjects on comparable movements prior to imagery testing. In Exps. 2- 4 this was accomplished by having separate groups of subjects participate in each experiment. Alternatively, for within-group designs imagery conditions could precede motor conditions (e.g. Exp. 1).

Manipulations similarly affect both imagery and movements. Manipulations that affect performance on movement control tasks should have comparable effects on imagery. For instance, grip (Exp. 3) and hand (Exp. 4) preferences in both PJ and MC conditions were shown to be similarly influenced by changes in the relative hand orientation of the stimulus. In both types of task, subjects consistently preferred less awkward grips.

Evidence of analog processing. Because motor imagery is by nature dynamic, 
there should be evidence of analog processing. That is, the time required to imagine a movement should be influenced by variables known to affect the duration of actual movements. In the present case PJs of awkwardness (Exp. 2), grip (Exp. 3b), and hand (Exp. 4b) preferences took longer when they would have involved more cumbersome, and thus slower, movements. In addition, the amount of time required for both grip and hand selection judgements increased as an approximately linear function of the number of degrees of hand rotation that would have been required to move from the hands' current resting positions into the selected posture.

Motor not visual imagery. Care should be taken to ensure that findings are not attributable to visual mental imagery. For example, the fact that RTs in Exps. 2b, 3b, and $4 \mathrm{~b}$ increased as a function of both the perceived awkwardness and extent of would-be movements, is only expected if mental simulations are sensitive to biomechanical constraints. By contrast, transformations of visual mental images are wellknown to follow the shortest path irrespective of such boundaries (e.g. Shepard \& Cooper, 1982).

Domain general solution. Because motor imagery is presumed to involve transforming somatomotor representations to imagine entire movements, the information made available from these simulations should be useful for solving many different types of movement-related problems. Consequently, results interpreted as reflecting use of motor imagery should be consistent across a variety of different tasks. Over all of the studies, response preferences in imagery and prehension were highly correlated and reflected the unique biomechanical properties of the left and right hands. Likewise, as mentioned above, RTs all tended to increase as a function of both the awkwardness and extent of the would-be movement.

Shared neural substrates. Finally, tasks involving motor imagery should engage areas of the brain known to be involved in somatomotor processing (for a comprehensive review see Jeannerod, 1994). Although not directly addressed in the present studies, earlier work using variations of the grip selection task in normals (Johnson, 1998), split-brain (Johnson et al., 1999), and hemiplegic patients (Johnson, 1999) indicates that PJs involve cerebral mechanisms used in motor planning and/or execution.

\section{Acknowledgements}

Preparation of this manuscript was supported in part by a grant from the James S. McDonnell Foundation to S.H.J. The author wishes to acknowledge Natascha Tokowicz, Erik Hawley, and Brad Wedemeyer for their assistance with data collection, and James E. Cutting, Michael McCarty, David A. Rosenbaum, and anonymous reviewers for helpful comments. Portions of this work were conducted while the author was at the University of Massachusetts at Amherst, and at Gettysburg College, and were presented at the 37th Annual Meeting of the Psychonomic Society, Chicago, IL. This paper is devoted to the memory of Dr John M. Rybash. 


\section{References}

Annett, J. (1995). Imagery and motor processes: editorial overview. British Journal of Psychology, 86, $161-167$.

Bernstein, N. (1967). The Coordination and Regulation of Movements, London: Pergamon.

Carello, C., Grosofsky, A., Reichel, F. D., Solomon, H. Y., \& Turvey, M. T. (1989). Visually perceiving what is reachable. Ecological Psychology, 1, 27-54.

Crammond, D. J. (1997). Motor imagery: never in your wildest dream. Trends in Neurosciences, 20, 5457.

Cutting, J. E. (1982). Two ecological perspectives: Gibson vs Shaw and Turvey. American Journal of Psychology, 95, 199-222.

Decety, J., \& Boisson, D. (1990). Effect of brain and spinal cord injuries on motor imagery. European Archives of Psychiatry and Clinical Neuroscience, 240, 39-43.

Decety, J., \& Jeannerod, M. (1995). Fitt's Law in mentally simulated movements. Behavioral Brain Research, 72, 127-134.

Decety, J., Philippon, B., \& Ingvar, D. H. (1988). rCBF landscapes during motor performance and motor ideation of a graphic gesture. European Archives of Psychiatry and Neurological Sciences, 238, 33 38.

Decety, J., Jeannerod, M., \& Prablanc, C. (1989). The timing of mentally represented actions. Behavioral Brain Research, 34, 35-42.

Decety, J., Perani, D., Jeannerod, M., Bettinardi, V., Tadary, B., Woods, R., Mazziotta, J. C., \& Fazio, F. (1994). Mapping motor representation with PET. Nature, 371, 600-602.

Deiber, M. -P., Ibanez, V., Honda, M., Sadato, N., Raman, R., \& Hallett, M. (1997). Cerebral processes related to visuomotor imagery and generation of simple finger movements studied with positron emmission tomography. Neuroimage, 7, 73-85.

Dominey, P., Decety, J., Broussolle, Chazot, G., \& Jeannerod, M. (1995). Motor imagery of a lateralized sequential task is asymmetrically slowed in hemi-Parkinson patients. Neuropsychologia, 33, 727-741.

Fitts, P. M. (1954). The information capacity of the human motor system in controlling the amplitude of movement. Journal of Experimental Psychology, 47, 381-391.

Gandevia, S. C. (1982). The perception of motor commands of effort during muscular paralysis. Brain, $105,151-159$.

Georgopoulos, A. P., \& Massey, J. T. (1987). Cognitive spatial-motor processes. Experimental Brain Research, 65, 361-370.

Georgopoulos, A. P., Lurito, J., Petrides, M., Schwartz, A. B., \& Massey, J. T. (1989). Mental rotation of the neuronal population vector. Science, 243, 234-236.

Gibson, J. J. (1979). The Ecological Approach to Visual Perception, Hillsdale, NJ: Erlbaum.

Hallett, M., Fieldman, J., Cohen, L. G., Sadato, N., \& Pascual-Leone, A. (1994). Involvement of primary motor cortex in motor imagery and mental practice. Behavioral and Brain Sciences, 17, 210.

Heft, H. (1993). A methodological note on overestimates of reaching distance: Distinguishing between perceptual and analytical judgements. Ecological Psychology, 5, 255-271.

Hildebrandt, H., \& Zeiger, A. (1995). Unconscious activation of motor responses in a hemiplegic patient with anosognosia and neglect. European Archives of Psychiatry and Clinical Neuroscience, 246, 5359.

James, W. (1890). Principles of Psychology, New York: MacMillan.

Jeannerod, M. (1994). The representing brain: Neural correlates of motor intention and imagery. Brain and Behavioral Sciences, 17, 187-245.

Jeannerod, M. (1995). Mental imagery in the motor context. Neuropsychologia, 33, 1419-1432.

Jiang, Y., \& Mark, L. S. (1994). The effect of gap depth on the perception of whether a gap is crossable. Perception and Psychophysics, 56, 691-700.

Johnson, S.H., Corballis, P.M., Gazzaniga, M.S., (1999). Within grasp but out of reach: evidence for a double dissociation between imagined hand and arm movements in the left cerebral hemisphere. Manuscript under review.

Johnson, S. H. (1991). Commentary on Tarr and Pinker. Psychological Science, 2, 205-206. 
Johnson, S. H. (1998). Cerebral organization of motor imagery: Contralateral control of grip selection in mentally represented prehension. Psychological Science, 9, 219-222.

Johnson, S.H., 1999. Imagining the impossible: Intact motor representations in hemiplegic patients. Manuscript under review.

Jolicoeur, P., \& Landau, M. J. (1984). Effects of orientation on the identification of simple visual patterns. Canadian Journal of Psychology, 38, 80-93.

Klatzky, R. L., McCloskey, B., Doherty, S., Pelligrino, J., \& Smith, T. (1987). Knowledge about hand shaping and knowledge about objects. Journal of Motor Behavior, 19, 187-213.

Klatzky, R. L., Pelligrino, J., McCloskey, B., \& Lederman, S. J. (1993). Cognitive representations of functional interactions with objects. Memory and Cognition, 21, 294-303.

Kohl, R. M., \& Fisicaro, S. A. (1996). Response intention and imagery processes: Locus, interaction, and contribution to motor learning. Brain and Behavioral Sciences, 19, 760-762.

Kosslyn, S. M. (1980). Image and Mind, Cambridge, MA: Harvard University Press.

Kosslyn, S. M. (1995). Image and Brain, Cambridge, MA: MIT Press.

Lurito, J. T., Georgakopoulos, T., \& Georgopoulos, A. P. (1991). Cognitive spatial-motor processes: 7. The making of movements at an angle from a stimulus direction: Studies of motor cortical activity at the single cell and population levels. Experimental Brain Research, 87, 562-580.

MacKenzie, C. L., \& Iberall, T. (1994). The Grasping Hand, New York: North-Holland.

Mark, L. S., \& Vogele, D. (1988). A biodynamic basis for perceived categories of action: A study of sitting and stair climbing. Journal of Motor Behavior, 19, 367-384.

Mark, L. S. (1995). Perceiving the preferred critical boundary for an affordance. In B. Bardy, R. Bootsma, \& Y. Guiard, Studies in perception and action III, (pp. 129-132). Hillsdale, NJ: Erlbaum.

Mark, L. S., Nemeth, K., Gardner, D., Dainoff, M. J., Paasche, J., Duffy, M., \& Grant, K. (1997). Postural dynamics and the preferred critical boundary for visually guided reaching. Journal of Experimental Psychology: Human Perception and Performance, 23, 1365-1379.

Marteniuk, R. G., MacKenzie, C. L., Jeannerod, M., Athenes, S., \& Dugas, C. (1987). Constraints on human arm movement trajectories. Canadian Journal of Psychology, 41, 365-378.

Parsons, L. M., Fox, P. T., Downs, J. H., Glass, T., Hirsch, T. B., Martin, C. C., Jerabek, P. A., \& Lancaster, J. L. (1995). Use of implicit motor imagery for visual shape discrimination as revealed by PET. Nature, 375, 54-58.

Parsons, L. M. (1987). Imagined spatial transformations of one's body. Journal of Experimental Psychology: Human Perception and Performance, 116, 172-191.

Parsons, L. M. (1987). Imagined spatial transformations of one's hands and feet. Cognitive Psychology, 19, 178-241.

Parsons, L. M. (1994). Temporal and kinematic properties of motor behavior reflected in mentally simulated action. Journal of Experimental Psychology: Human Perception and Performance, 20, 709-730.

Pellizzer, G., \& Georgopoulos, A. P. (1993). Common processing constraints for visuomotor and visual mental rotations. Experimental Brain Research, 93, 165-172.

Pfurtscheller, G., \& Neuper, C. (1997). Motor imagery activates primary sensorimotor area in humans. Neuroscience Letters, 239, 65-68.

Rao, S. M., Binder, J. R., Bandettini, P. A., Hammeke, T. A., Yetkin, F. Z., Jesmanowicz, A., Lisk, L. M., Morris, G. L., Mueller, W. M., Estkowski, R. T. R., Wong, E. C., Haughton, V. M., \& Hyde, J. S. (1993). Functional magnetic resonance of complex human movements. Neurology, 43, 2311-2318.

Robinovitch, S. N., \& Cronin, T. (1997). Age-related changes in perceptions of postural stability. Society for Neuroscience, 23, 1140.

Roland, P. E., Skinhoj, E., Lassen, N. A., \& Larsen, B. (1980). Different cortical areas in man in organization of voluntary movements in extrapersonal space. Journal of Neurophysiology, 43, 137150.

Rosenbaum, D. A., \& Jorgenson, M. J. (1990). Planning macroscopic aspects of manual control. Human Movement Science, 11, 61-69.

Rosenbaum, D. A., Vaughan, J., Barnes, H. J., \& Jorgensen, M. J. (1992). Timecourse of movement planning: Selection of handgrips for object manipulation. Journal of Experimental Psychology: Learning, Memory, and Cognition, 18, 1058-1073. 
Rosenbaum, D. A. (1991). Human Motor Control, New York: Academic Press.

Roth, M., et al. (1996). Possible involvement of primary motor cortex in mentally simulated movement: a functional magnetic resonance imaging study. Neuroreport, 7, 1280-1284.

Shepard, R. N., \& Cooper, L. A. (1982). Mental Images and Their Transformations, Cambridge, MA: MIT Press.

Sirigu, A., Cohen, L., Duhamel, J. R., Pillon, B., Dubois, B., Agid, Y., \& Pierrot-Deseilligny, C. (1995). Congruent unilateral impairments for real and imagined hand movements. Neuroreport, 6, 997-1001.

Sirigu, A., Duhamel, J. -R., Cohen, L., Pillon, B., Dubois, B., \& Agid, Y. (1996). The mental representation of hand movements after parietal cortex damage. Science, 273, 1564-1568.

Stelmach, G. E., Castiello, U., \& Jeannerod, M. (1994). Orienting the finger opposition space during prehension movements. Journal of Motor Behavior, 26, 178-186.

Stephan, K. M., Fink, G. R., Passingham, R. E., Silbersweig, D., Ceballos-Baumann, A. O., Frith, C. D., \& Frackowiak, R. S. J. (1995). Functional anatomy of the mental representation of upper extremity movements in healthy subjects. Journal of Neurophysiology, 73, 373-386.

Tarr, M. J., \& Pinker, S. (1990). When does human object recognition use a viewer-centered reference frame? Psychological Science, 1, 253-256.

Tarr, M. J. (1995). Rotating objects to recognize them: A case study on the role of viewpoint dependency in the recognition of three-dimensional objects. Psychonomic Bulletin and Review, 2, 55-82.

Warren, W. H. (1984). Perceiving affordances: Visual guidance of stair climbing. Journal of Experimental Psychology: Human Perception and Performance, 13, 371-383.

Weiss, T., Hansen, E., Rost, R., \& Beyer, L. (1994). Mental practice of motor skills used in poststroke rehabilitation has its own effects on central nervous activation. International Journal of Neuroscience, $78,157-166$.

Wexler, M., Kosslyn, S. M., \& Berthoz, A. (1998). Motor processes in mental rotation. Cognition, 68, 7794. 